\title{
Chiral Spin Liquid Phase of the Triangular Lattice Hubbard Model: A Density Matrix Renormalization Group Study
}

\author{
Aaron Szasz $\oplus^{1,2,3, *}$ Johannes Motruk, ${ }^{1,2}$ Michael P. Zaletel, ${ }^{1,2,4}$ and Joel E. Moore ${ }^{1,2}$ \\ ${ }^{1}$ Department of Physics, University of California, Berkeley, California 94720, USA \\ ${ }^{2}$ Materials Sciences Division, Lawrence Berkeley National Laboratory, Berkeley, California 94720, USA \\ ${ }^{3}$ Perimeter Institute for Theoretical Physics, Waterloo, Ontario N2L 2Y5, Canada \\ ${ }^{4}$ Department of Physics, Princeton University, Princeton, New Jersey 08540, USA
}

(Received 8 August 2019; revised manuscript received 4 December 2019; accepted 18 March 2020; published 22 May 2020)

\begin{abstract}
Motivated by experimental studies that have found signatures of a quantum spin liquid phase in organic crystals whose structure is well described by the two-dimensional triangular lattice, we study the Hubbard model on this lattice at half filling using the infinite-system density matrix renormalization group (iDMRG) method. On infinite cylinders with finite circumference, we identify an intermediate phase between observed metallic behavior at low interaction strength and Mott insulating spin-ordered behavior at strong interactions. Chiral ordering from spontaneous breaking of time-reversal symmetry, a fractionally quantized spin Hall response, and characteristic level statistics in the entanglement spectrum in the intermediate phase provide strong evidence for the existence of a chiral spin liquid in the full twodimensional limit of the model.
\end{abstract}

DOI: 10.1103/PhysRevX.10.021042

Subject Areas: Condensed Matter Physics, Magnetism, Strongly Correlated Materials

\section{INTRODUCTION}

Quantum spin liquids [1-3] have been the subject of considerable interest since the concept was first introduced in 1973 by Anderson, who suggested that geometrical frustration on the triangular lattice could lead to a resonating valence bond ground state of the antiferromagnetic Heisenberg model [4]. Although it is now known that the Heisenberg model on the triangular lattice in fact exhibits a three-sublattice $120^{\circ}$ order in the ground state $[5,6]$, antiferromagnetic models on the triangular lattice remain some of the most promising systems to realize a phase in which spins remain disordered even down to zero temperature. The triangular lattice has seemed particularly promising since the work of Shimizu et al., who found that the organic crystal $\kappa$-(BEDT-TTF) ${ }_{2} \mathrm{Cu}_{2}(\mathrm{CN})_{3}$, which is well described by independent 2D layers with nearly isotropic triangular lattice structure, shows no sign of spin ordering even down to tens of $\mathrm{mK}$, indicative of a possible spin liquid ground state [7]. Subsequent studies of this crystal have found that the heat capacity is $T$-linear at low temperature [8], suggesting the presence of low-lying

\footnotetext{
*aszasz@perimeterinstitute.ca
}

Published by the American Physical Society under the terms of the Creative Commons Attribution 4.0 International license. Further distribution of this work must maintain attribution to the author(s) and the published article's title, journal citation, and DOI. gapless excitations, but also that the thermal conductivity has no such $T$-linear contribution [9], indicating to the contrary that there is a gap in the energy spectrum. Another triangular lattice material, $\mathrm{EtMe}_{3} \mathrm{Sb}\left[\mathrm{Pd}(\mathrm{dmit})_{2}\right]_{2}$, was until recently believed to show $T$-linear behavior in both the heat capacity and thermal conductivity [10-13], but new experiments show that it too may exhibit gapped thermal transport [14-16]. The true nature of the spin liquid phases in these and other triangular lattice materials [17-19] such as $\mathrm{YbMgGaO}_{4}[20,21]$ remains unclear.

Substantial theoretical effort has gone into answering this question, primarily in studying the antiferromagnetic Heisenberg model with additional terms, such as secondneighbor interactions and ring exchanges, that frustrate the expected three-sublattice order [22-34]. The Heisenberg model and its extensions are derived from a perturbative expansion of a model of itinerant electrons, the Hubbard model [35]; by studying the Hubbard model directly, we can capture additional effects that may be important in actual materials, at the cost of increased computational effort-compared with spin-1/2 models, the size of the local Hilbert space is doubled, so the system sizes that can be accessed by full-Hilbert-space numerical methods are only about half as large.

Although there is now a wide variety of theoretical evidence pointing to the existence of a nonmagnetic insulating phase of the triangular lattice Hubbard model [22,36-47], there is still little agreement on the precise nature of the phase. Some candidates, suggested by results 
on both the Hubbard and extended Heisenberg models, include a $U(1)$ spin liquid with a spinon Fermi sea $[22,23,25,27,41,45]$, a nodal spin liquid [26,43], a gapped chiral spin liquid [28,48-51], and a $\mathbb{Z}_{2}$ spin liquid [28,29]. In this work, we confirm the existence of a nonmagnetic insulating phase of the Hubbard model on the triangular lattice at half filling, provide strong evidence that it is a gapped chiral spin liquid, and comment on possible experimental signatures.

We study the triangular lattice Hubbard model on infinite cylinders with finite circumference using the density matrix renormalization group (DMRG) technique [52-55], a variational method to find the ground state of a Hamiltonian within the matrix product state (MPS) ansatz. This method has previously been applied to an extended Hubbard model on a triangular lattice two-leg ladder, providing evidence for a $U(1)$ spin liquid phase with a spinon Fermi surface [45]. For systems larger than the twoleg ladder, to our knowledge there exists only one prior paper [47] that uses DMRG to study the triangular lattice Hubbard model. The authors of that study use the finite-system DMRG to confirm the existence of a nonmagnetic insulating phase. In our infinite-system DMRG study, we investigate the nature of the phase by studying the entanglement spectrum and the response to adiabatic spin-flux insertion through the cylinder as accomplished by twisting boundary conditions; we also study the MPS transfer matrix spectra, allowing us to rule out the possibility that we observe a Dirac spin liquid. We study the model on a variety of cylinders with different circumferences and boundary conditions. With some cylinder geometries we find a chiral spin liquid phase regardless of how we twist the boundary conditions, while for the others the chiral phase exists for a range of twisted boundary conditions, in particular for those boundary conditions for which the ground state is closest to obeying the symmetries of the full two-dimensional lattice. While caution is required when extrapolating from cylinders to the 2D limit, taken together, the results for the various cylinders point to the existence of the chiral spin liquid phase in the full two-dimensional lattice as well.

The organization of the paper is as follows: In Sec. II, we introduce the model we study and the mixed-space representation used in the simulations. In Sec. III, we demonstrate the existence of metallic, nonmagnetic insulating, and magnetically ordered phases of the model and, furthermore, show that the intermediate phase breaks time-reversal symmetry. We present detailed results for five different cylinder geometries. Readers wishing to see even more complete data are encouraged to read the Supplemental Material [56]; those interested primarily in the identification of the chiral spin liquid phase can proceed to Sec. IV, in which we show that the intermediate phase is, in fact, a chiral spin liquid. Finally, in Sec. V, we discuss the results, placing them in the context of recent experiments and other theoretical studies.

\section{THE MODEL}

The model we study is the standard Hubbard Hamiltonian,

$$
H=-t \sum_{\langle i j\rangle \sigma} c_{i \sigma}^{\dagger} c_{j \sigma}+\text { H.c. }+U \sum_{i} n_{i \uparrow} n_{i \downarrow}
$$

where $c_{i \sigma}\left(c_{i \sigma}^{\dagger}\right)$ is the fermion annihilation (creation) operator for spin $\sigma$ on site $i$ and $n=c^{\dagger} c$ is the number operator; $\langle\cdot\rangle$ indicates nearest-neighbor pairs on the triangular lattice (Fig. 1). We work at half filling with net zero spin, so that $\sum_{i}\left\langle n_{i \uparrow}\right\rangle=\sum_{i}\left\langle n_{i \downarrow}\right\rangle=N / 2$, where $N$ is the total number of sites. This model has a single tunable parameter, $U / t$. In the limit $U=0$, the model is exactly solvable and at half filling forms a metal with a nearly circular Fermi surface; in the limit $U \rightarrow \infty$, double occupancy is disallowed, so to lowest order in perturbation theory in $t / U$, the model reduces to the nearest-neighbor antiferromagnetic Heisenberg model [35], whose ground state exhibits a three-sublattice spin order $[5,6]$. Between these two limits of $U=0$ and $U \rightarrow \infty$, there must be at least one phase transition, from the metallic to the Mottinsulating phase; it is in the vicinity of this metal-insulator transition that a spin liquid phase is likely to be found.

To study this model using the DMRG method, we wrap the two-dimensional triangular lattice onto an infinitely long cylinder of finite circumference. We primarily use the so-called YC boundary conditions [56,57], for which the triangles are oriented such that one of the sides runs along (a)

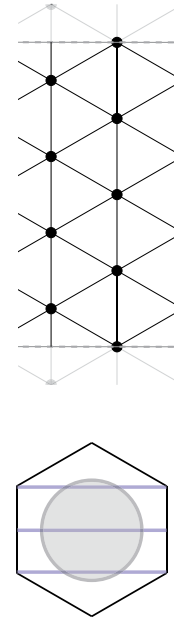

(c)

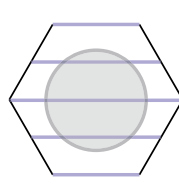

(d)

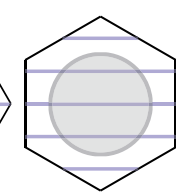

(e) (b)

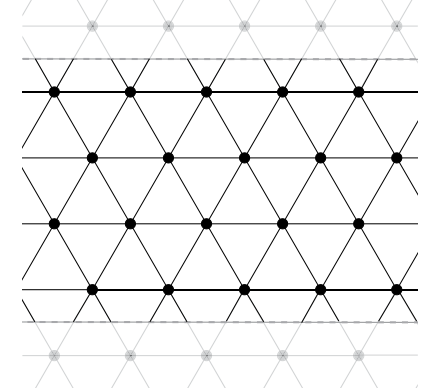

FIG. 1. (a) Triangular lattice on a cylinder of circumference 4 with YC boundary conditions (YC4 cylinder); the dashed lines are identified together and run along the length of the cylinder. (b) XC4 cylinder. (c)-(g) Horizontal lines show allowed momenta in the Brillouin zone for the $\mathrm{YC} 3, \mathrm{XC} 4, \mathrm{YC} 4, \mathrm{YC} 5$, and $\mathrm{YC} 6$ cylinders, in order of increasing circumference. The shaded circle shows the Fermi surface for noninteracting electrons $(U=0)$. 
the circumference of the cylinder. The YC4 lattice is shown in Fig. 1(a) as an example, with the dashed gray lines identified together with periodic boundaries to form a cylinder. We also consider XC boundary conditions, for which one triangle side runs along the length of the cylinder. We show the XC4 lattice in Fig. 1(b); an XCn cylinder, which exists only for even $n$, has a physical circumference of $n \sqrt{3} / 2$ lattice constants.

Denoting translation by one lattice constant around the cylinder by $T_{y}$, the $\mathrm{YCn}$ cylinder has a discrete translation symmetry $T_{y}^{n}=1$; we explicitly conserve the momentum quantum numbers associated with this symmetry by rewriting the Hamiltonian in a mixed real- and momentum-space basis with single-particle operators $c_{x, k_{y}, \sigma}$, which both gives substantial improvements in computational efficiency and allows us to separately find the ground state in different momentum sectors [58,59]. Similarly, for the XCn cylinders we define the translation operator $T_{y}^{\mathrm{XC}}$ that translates between two-site unit cells around the circumference, with $\left(T_{y}^{\mathrm{XC}}\right)^{n / 2}=1$; we can again exploit momentum conservation but with only half as many quantum numbers.

In this paper, we particularly focus on the YC4 and YC6 cylinders, and we also present and discuss data for the YC3, $\mathrm{XC} 4$, and YC5 cylinders. For the various cylinders, the finite circumferences and periodic boundary conditions restrict the accessible momenta in the Brillouin zone as shown in Figs. 1(c)-1(g).

\section{PHASE DIAGRAM}

Our goal is to show that the Hubbard model on the full two-dimensional triangular lattice has a chiral spin liquid phase; we begin by establishing the phase diagram more generally, showing the existence of the expected metallic, nonmagnetic insulating (NMI), and magnetic phases, and we furthermore show that the NMI phase breaks timereversal symmetry.

Of course, we have access in our simulations not to the full two-dimensional model but rather to a collection of finite circumference cylinders. To overcome this impediment, we employ three methods: (i) each phase that exists in the two-dimensional model should leave characteristic signatures when restricted to a finite circumference cylinder, and we can look for these signatures; (ii) for each cylinder, we can twist the boundary conditions to scan the allowed momentum cuts [Figs. 1(c)-1(g)] through the full two-dimensional Brillouin zone; and (iii) we can compare the results for the various cylinders and look for trends and commonalities. The third is self-explanatory; before presenting the data, we elaborate on (i) and (ii).

We first discuss how the various possible phases of the two-dimensional model should manifest on the infinite cylinders we study. A metallic state will be gapless, as indicated by a nonzero value for the central charge $c$ of the one-dimensional conformal field theory corresponding to the restriction of the two-dimensional model to the onedimensional allowed momentum cuts; in particular, if the Fermi surface intersects $N_{F}$ of the allowed momentum lines in the Brillouin zone [see Figs. 1(c)-1(g)], the central charge will be $c=2 N_{F}[56,60]$. We expect the $120^{\circ}$ magnetically ordered phase to be fully gapped $(c=0)$ and symmetric on even circumference cylinders due to the integer-spin Haldane gap [61] induced by the reduced dimension but gapless on odd circumference cylinders; the 2D spin order should qualitatively manifest as large peaks in the spin structure factor at the $K^{ \pm}$points which diverge linearly with cylinder circumference. If the intermediate phase is a $U(1)$ spin liquid with a spinon Fermi surface, there will be a charge gap but no spin gap, leading to cylinder central charge $c=2 N_{F}-1$ and $2 k_{F}$-singularities in the structure factors $[23,45,62,63]$. Finally, a gapped spin liquid will have $c=0$ and feature several "topologically degenerate" low-lying states whose energy splitting decreases exponentially with circumference [64], along with other topological signatures we will return to in detail. The chiral spin liquid in particular will spontaneously break time-reversal and parity symmetry while retaining all others; time-reversal symmetry breaking is indicated by a nonzero scalar chiral order parameter $\left\langle\mathbf{S}_{i} \cdot\left(\mathbf{S}_{j} \times \mathbf{S}_{k}\right)\right\rangle$, where $i, j$, and $k$ label the vertices of a triangle in the lattice [65]. In the simulations, all these properties must be assessed as a function of the DMRG accuracy as captured by the bond dimension $\chi$ of the MPS ansatz.

We next discuss how, for a given cylinder geometry, twisting of boundary conditions grants access to the full two-dimensional Brillouin zone. In particular, instead of using periodic boundaries $c_{x, y=0, \sigma}=c_{x, y=L, \sigma}$, we set $c_{x, y=0, \sigma}=e^{i \theta \sigma / 2} c_{x, y=L, \sigma}$, followed by the gauge transformation $c_{x, y, \sigma} \mapsto e^{i \theta \sigma y /(2 L)} c_{x, y, \sigma}$, where $\sigma$ in the exponent is +1 for spin up and -1 for spin down. Physically, this is equivalent to inserting a flux through the cylinder of $\theta / 2$ for spin-up electrons and $-\theta / 2$ for spin down, which corresponds to flux $\theta$ for the spin degrees of freedom. Note that, because the flux insertion is opposite for spin up and spin down, this transformation does not break time-reversal symmetry.

When the original Hamiltonian with periodic boundaries is written in the mixed-space picture, some coefficients will depend on the momentum $k$ around the cylinder; the only effect of the flux insertion is to transform those coefficients, with $k=(2 \pi / L) n \mapsto(2 \pi / L)(n+\theta \sigma / 2)$. This transformation can be viewed as shifting the momentum cuts in the Brillouin zone, upward for spin up and downward for spin down, as illustrated in Fig. 2. Thus, by scanning $\theta$ from 0 to $4 \pi$, we can access the full two-dimensional Brillouin zone, giving substantial additional evidence for the two-dimensional model despite using only a single cylinder geometry.

The only physical effect of this flux insertion is from the twisted boundary conditions, and in the two-dimensional limit where the cylinder circumference becomes infinitely 


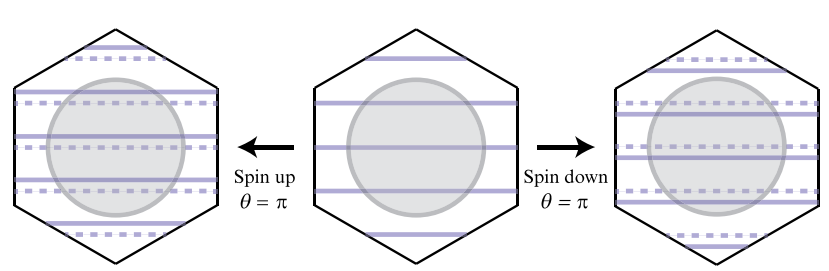

FIG. 2. The effect of flux insertion on the mixed-space model is to shift the allowed momentum cuts through the Brillouin zone. They shift upward for spin-up electrons and downward for spindown electrons, thus preserving time-reversal symmetry. Note that for $\theta=4 \pi n$ for any integer $n$, the cuts are again in their original positions.

large, the effect on local properties like order parameters and short-range correlation functions must go to zero. Thus the variation in these quantities with flux insertion serves as an indication of the degree of "two-dimensionality" of the cylinders we study and therefore of the reliability of our results in predicting the behavior of the full twodimensional model.

Note that the flux insertion can be performed adiabatically by first computing the ground state with periodic boundary conditions and then increasing $\theta$ in small increments, at each step using the converged ground state from the previous step as the initial state for the new simulation. Notably, this procedure allows for detection of spin pumping from a quantized spin Hall effect, which is a hallmark of the chiral spin liquid phase.

We now present results for the various cylinder geometries we have studied.

\section{A. YC4}

Out of the five different cylinders we consider, our most extensive data are for YC4, which strikes a balance between two-dimensionality (favoring larger cylinders) and ability to converge the DMRG simulations (favoring smaller ones).

On the YC4 cylinder with periodic boundaries we find three phases, corresponding to the expected metallic, nonmagnetic insulating (NMI), and spin-ordered phases of the full two-dimensional model; the phase diagram is summarized in Fig. 3, along with our results for several physical quantities: the correlation length, spin structure factor, and scalar chiral order parameter.

The transition from the NMI phase to the spin-ordered phase at $U / t \approx 10.6$ is indicated by a peak in the correlation length, the appearance of large peaks near the $K^{ \pm}$points of the Brillouin zone in the spin structure factor, and the vanishing of the chiral order parameter. The spin structure factor in particular allows us to identify the high- $U$ side of this transition as the one-dimensional descendant of the two-dimensional magnetically ordered phase, and the intermediate- $U$ side as nonmagnetic.

Because the metal is gapless, the metal to NMI transition $(U / t \approx 8)$ is less clear from the direct physical
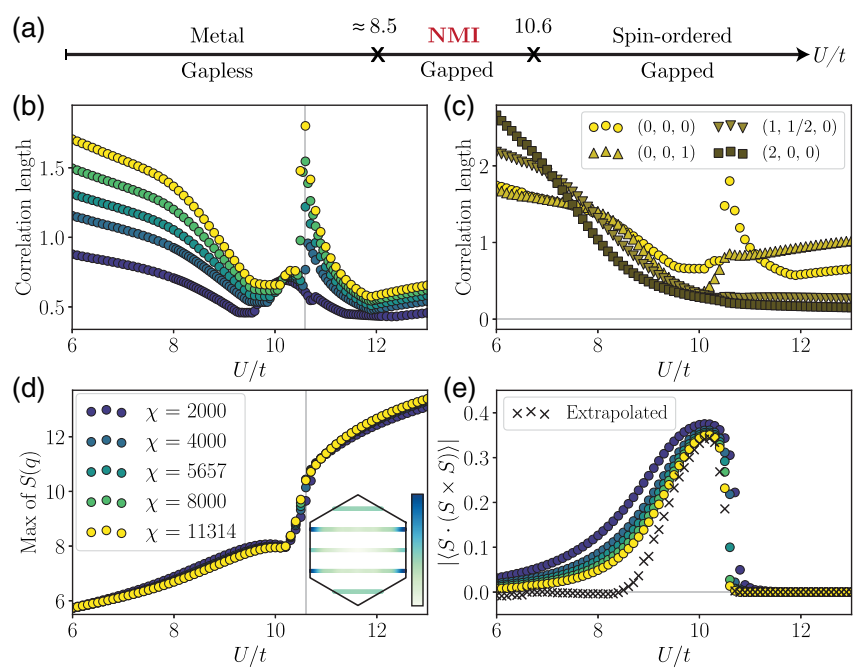

FIG. 3. Results for the YC4 cylinder. Results are shown for a range of MPS bond dimensions $\chi$ as indicated in the lower left legend. (a) A nonmagnetic insulating (NMI) phase appears between a gapless metallic phase at low $U / t$ and a magnetic phase at high $U / t$. (b) Correlation length in the "charge neutral sector," in other words for excitations carrying no charge, spin, or momentum. The vertical line at $U / t=10.6$ is provided as a guide to the eye. (c) Correlation lengths at the largest bond dimension in various charge sectors. The sector $(Q, S, K)$ corresponds to correlations $\left\langle\mathcal{O}_{1} \mathcal{O}_{2}\right\rangle$, where $\mathcal{O}_{1}$ creates and $\mathcal{O}_{2}$ annihilates an excitation carrying charge $Q$, spin $S$, and momentum quantum number $K$. (d) Spin structure factor: the curve shows the maximum value of the spin structure factor in the Brillouin zone. The inset shows the spin structure factor in the high- $U$ phase, with peaks at the closest allowed momenta to the $K^{ \pm}$ points, where they would be expected for $120^{\circ}$ magnetic ordering. Note that spin expectation values are reported here and throughout the paper with $\hbar / 2=1$. (e) Chiral order parameter $\left\langle\mathbf{S}_{i} \cdot\left(\mathbf{S}_{j} \times \mathbf{S}_{k}\right)\right\rangle$, where $i, j$, and $k$ label the three vertices of a triangle in the lattice, with an additional line showing extrapolation in the DMRG truncation error [66]; see Supplemental Material [56] for details on the extrapolation.

measurements shown in Fig. 3, though it is visible from the chiral order parameter: although a nonzero value of the order parameter indicates time-reversal symmetry breaking in both the metallic and NMI phases for finite bond dimension, an extrapolation in the DMRG truncation error [66] shows that the symmetry is actually unbroken in the low- $U$ phase [56]. To make this transition clearer, and to show that it corresponds specifically to the opening of a charge gap, we consider two additional quantities that we measure indirectly from the wave function via more involved calculations: the small- $k$ curvature of the densitydensity structure factor and the fermion quasiparticle weight; these are shown in Fig. 4.

The density-density structure factor $N(\mathbf{k})$ shows the presence or absence of a charge gap: if the system is gapless, for small momentum $N(\mathbf{k}) \propto|k|$, while if it is gapped, $N(\mathbf{k}) \propto k^{2}[67,68]$. Indeed, we find that at low $U$ 

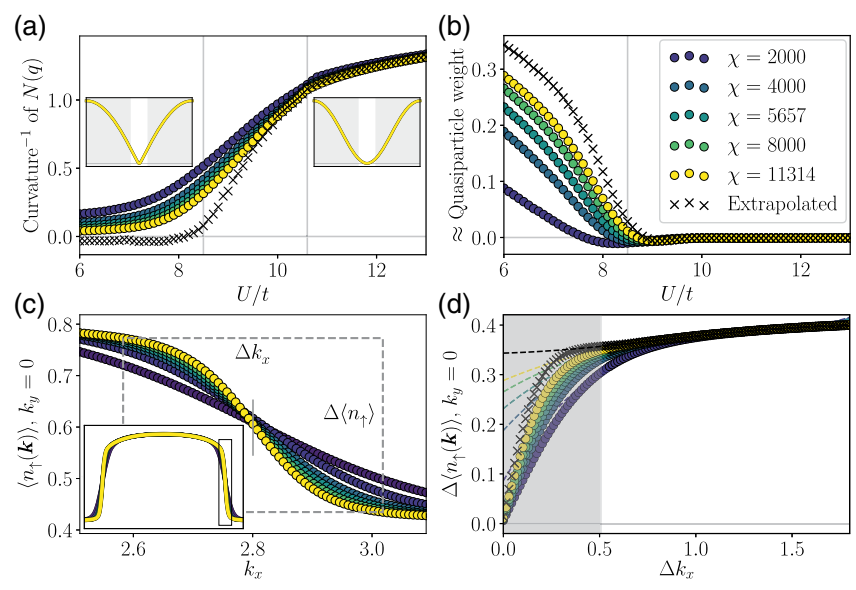

FIG. 4. Measurements pertaining to the metal-insulator transition for the YC4 cylinder. (a) Inverse curvature of the densitydensity structure factor at $k=0$, a proxy for the charge gap, with vertical lines at $U / t=8.5$ and 10.6 indicating the estimated locations of phase transitions. The insets show that the structure factor is linear around $k=0$ for small $U / t$ and quadratic at large $U / t$, indicating gapless and gapped phases, respectively; curvature is measured using a small interval around $k=0$, the nonshaded region in the insets. (b) Approximate fermionic quasiparticle weight, measured by discontinuity in occupation at the Fermi surface. The vertical line is at $U / t=8.5$. As described in the text, this is an indirect calculation rather than a direct measurement; the calculation is illustrated in the next two panels for one value of $U / t$, with further examples available in the Supplemental Material [56]. (c) Occupation of spin-up electrons as a function of $k_{x}$, with $k_{y}=0$, for $U / t=6$, both for the full Brillouin zone (inset) and for the edge of the Fermi surface, identified as the point with the steepest slope. The gap in occupation $\Delta\langle n\rangle$ can be measured as a function of the size $\Delta k_{x}$ of an interval symmetric around the Fermi surface. The result is shown in the next panel. (d) Change in occupation $\Delta\langle n\rangle$ across an interval $\Delta k_{x}$ around the Fermi surface. Small $\Delta k$ results are not reliable because of Luttinger liquid effects from the onedimensionality of the cylinder and because occupations are calculated by a finite range of real-space correlations. We therefore eliminate the points in the shaded region and fit the remainder with a cubic polynomial, then extrapolate to $\Delta k_{x}=0$ as shown by the dashed lines. These extrapolated results are the data shown in (b).

the structure factor is visibly linear near $k=0$, shown in Fig. 4(a) (left inset), while at high $U$ it is clearly quadratic (right inset). To observe the metal-insulator transition, we measure the curvature of the structure factor at $k=0$ and then extrapolate in the bond dimension. The extrapolated data reveal a transition from linear to quadratic at $U / t \approx 8.5$, consistent with the extrapolated chiral order parameter.

In Fig. 4(b), we show an estimate of the fermion quasiparticle weight, measured via the change in electron occupation at the Fermi surface. This calculation requires accounting for three different effects: (i) high resolution in momentum-space occupation requires very long-range correlation function calculations in real space; (ii) because a cylinder is a one-dimensional system, in a metallic phase it will behave as a Luttinger liquid and not actually have a discontinuity at the Fermi surface, though there is still a singularity; and (iii) finite bond dimension removes the singularity at the Fermi surface. The latter is easily dealt with by extrapolation; in the Supplemental Material [56] we show that the singularity reappears in the infinite bond dimension limit. The first effect is accounted for by measuring the change in occupation across a finite interval in $k$ symmetric around the Fermi surface. As long as the interval is sufficiently wide, the corresponding wavelength is short enough to not be affected by the finite range of the computed real-space correlations. The behavior above this cutoff in $\Delta k$ can then be extrapolated to $\Delta k=0$. This procedure is illustrated for $U / t=6$ in Figs. 4(c) and 4(d) and for other values of $U / t$ in the Supplemental Material [56].

The second effect cannot be accounted for rigorously using just the YC4 cylinder, since there is no well-defined quasiparticle weight in a quasi-one-dimensional system; in particular, all $\Delta k \lesssim 2 \pi / L=\pi / 2$ will be affected by the one-dimensionality of the cylinder, and this range includes approximately all $\Delta k$ around the Fermi surface out to the edge of the Brillouin zone. However, as can be seen in Fig. 4(d), the change in occupation does not show a qualitative change between $\Delta k=\pi / 2$ and the cutoff $\Delta k \gtrsim$ 0.5 used in our extrapolation, so the procedure described above and shown in Figs. 4(c) and 4(d) should still give an approximately correct estimate of the quasiparticle weight.

The resulting estimate of the quasiparticle weight appears to vanish around $U / t=9$, though there is some dependence on the precise form of extrapolation used. Since in computing the estimate we use only larger $\Delta k$ values, where there is a larger occupation gap, the actual quasiparticle weight should vanish at a slightly lower value of $U / t$, again being consistent with the chiral order parameter and structure factor curvature.

Further information about the three phases and the transitions between them comes from studying the entanglement in the system, and in particular from finite entanglement scaling [69-71]. If we cut the infinite cylinder into two semi-infinite halves, we can calculate the entanglement entropy $S$ between them from the eigenvalues $\lambda_{i}^{2}$ of the reduced density matrix of either side of the cut:

$$
S \equiv-\sum_{i} \lambda_{i}^{2} \log \left(\lambda_{i}^{2}\right)
$$

In the true ground state this is an infinite sum; however, when running DMRG simulations the MPS bond dimension $\chi$ upper bounds the number of nonzero $\lambda_{i}$ in Eq. (2) and thereby bounds $S \leq \log (\chi)$. In a gapless state, the true $S$ is infinite, as is the correlation length $\xi$, but finite entanglement scaling predicts that the two quantities will scale with $\chi$ such that [72]

$$
S \approx(c / 6) \log (\xi)
$$



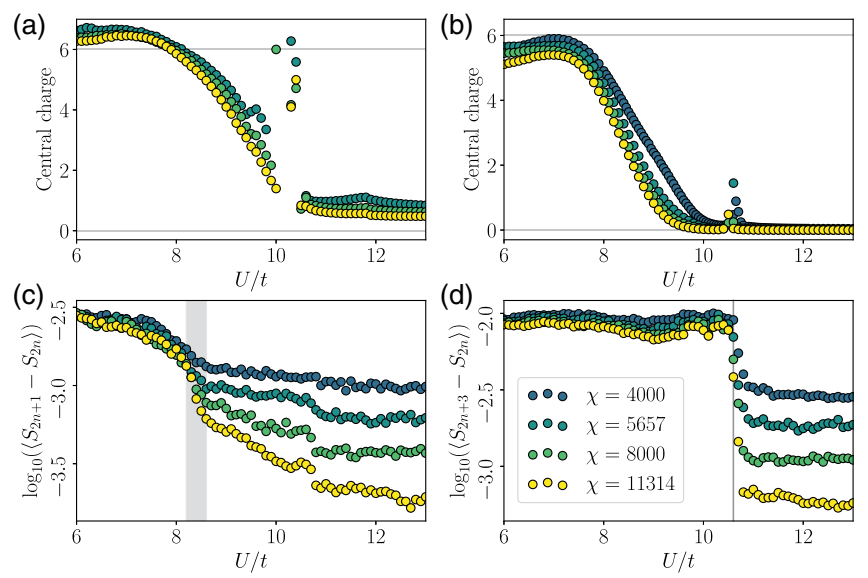

FIG. 5. Entanglement results for the YC4 cylinder. (a) Central charge of the effective one-dimensional state as calculated by the scaling of entanglement with correlation length, Eq. (3); this is the most accurate method for gapless systems. (b) Central charge as calculated by the scaling of entanglement with bond dimension, Eq. (4); this is the most accurate method for gapped systems. (c) Average separation between pairs of levels from the entanglement spectrum; the shaded region is $U / t=8.2-8.6$, where the spectrum approaches exact twofold degeneracy with increasing bond dimension. (d) Average separation among groups of four levels from the spectrum; the vertical line at $U / t=10.6$ marks the onset of fourfold degeneracy.

which can be used to estimate the central charge $c$ of the conformal field theory corresponding to the gapless metallic phase. We show the central charge computed using Eq. (3) in Fig. 5(a).

In a gapped state, $S$ is finite [73,74], so the DMRG estimate of $S$ should converge as $\chi$ is increased; however, $\xi$ will also converge, and the two quantities may converge at different rates so that the relative scaling between them becomes less reliable. In such a case, the central charge can be more accurately computed by direct scaling of entanglement with bond dimension [69-71]:

$$
S \approx(1+\sqrt{12 / c})^{-1} \log (\chi)
$$

We show the central charge computed using Eq. (4) in Fig. 5(b).

Until $U / t \approx 8$, the central charge is constant with respect to $U / t$ and is near to the value $c=6$ that we would expect for a metallic state [56,60]. For $U / t \gtrsim 9$, it is clear from Fig. 5(b) that $c=0$, indicating that the phases are gapped. For intermediate values of $8 \lesssim U / t \lesssim 9$, the central charge is still far from converged with bond dimension, but it is plausible that it will extrapolate to zero; see Supplemental Material for details [56]. Note that the apparently unsystematic behavior in Fig. 5(a) near the previously identified transition at $U / t \approx 10.6$ is due to a slight shift in the location of the peak in the correlation length as bond dimension is increased.

We can identify the locations of both phase transitions with more precision by studying the entanglement spectrum, which is the list of values $\left\{-\log \left(\lambda_{i}\right)\right\}$, for the same $\left\{\lambda_{i}\right\}$ appearing in Eq. (2); the full low-lying entanglement spectrum is shown as a function of $U / t$ in the Supplemental Material [56]. In particular, by pairing the levels and then finding the average separation between the levels in each pair, we observe that the entire spectrum acquires an exact twofold degeneracy for $U / t \gtrsim 8.4$; this is shown in Fig. 5(c). Although at finite bond dimension there remains for any $U / t$ a finite separation between the levels in each pair, for $U / t<8.2$ the separation does not visibly depend on the bond dimension, whereas for $U / t \geq 8.6$, the separation goes to 0 with increasing bond dimension; the exact location of the transition is not clear from our data but appears to be in the range of $8.2 \lesssim U / t \lesssim 8.6$, which is consistent with the location of the metal-insulator transition as found above. We can similarly group the entanglement spectrum into sets of four levels and consider the average separation of the highest and lowest levels in each group, revealing the onset of fourfold degeneracy at $U / t \approx 10.6$, at the spin-ordering transition. This fourfold degeneracy corresponds to the different projective representations of the symmetry group carried by the entanglement spectrum [75].

Taken together, the data in Figs. 3-5 demonstrate that the YC4 cylinder with periodic boundary conditions exhibits three distinct phases, corresponding to metallic, timereversal symmetry-breaking nonmagnetic insulating, and magnetically ordered phases in two dimensions. The nature of the transitions is a more challenging question. The metalinsulator transition appears to be continuous. No quantity we measure, including correlation length, spin order, chiral order, estimate of the quasiparticle weight, and the entanglement spectrum, shows any kind of discontinuity; even in a weakly first-order transition, we would expect to see some such signature in our data. However, despite the very large bond dimensions we use, we are unable to pinpoint the location of the transition, so further properties of the metalinsulator transition such as critical exponents are not feasible to calculate. The magnetic ordering transition shows much more abrupt changes in various quantities, especially the chiral order parameter, though none are clearly discontinuous. There may be a very small discontinuity in the entanglement spectrum (Fig. S23 in the Supplemental Material [56]), which could indicate a weakly first-order transition, but much higher bond dimension would be required to make a definitive statement. To summarize, the metal-insulator transition appears to be continuous, while the spin-ordering transition is either continuous or very weakly first order.

We now turn to the results of flux insertion. We perform the flux insertion adiabatically, twisting the boundary conditions in intervals of $\theta=\pi / 12$. Because of the much larger parameter space spanned by both $U / t$ and $\theta$, we restrict our computations to a single bond dimension, $\chi=4000$. Based on the data shown in Fig. 3, we believe 

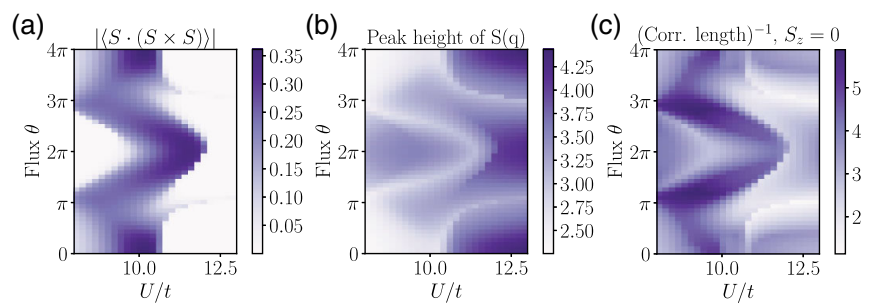

FIG. 6. YC4 cylinder with flux insertion $\theta$, for $\chi=4000$. (a) Absolute value of chiral order parameter. The chiral phase exists for all twisted boundary conditions, but the phase boundaries shift with $\theta$. (b) Maximum of $\left\langle S_{z} S_{z}\right\rangle$ structure factor on allowed momentum cuts in the Brillouin zone. (c) Inverse correlation length for excitations with $S_{z}=0$, which serves as a proxy for the spin-singlet gap.

this bond dimension is sufficient to capture the qualitative behavior of the system; furthermore, we increase the bond dimension to 8000 for several values of $U / t$ and confirm that there is no qualitative change.

In Fig. 6, we show several quantities computed as a function of both $U / t$ and $\theta$, namely the chiral order parameter, the maximum value of the $\left\langle S_{z} S_{z}\right\rangle$ structure factor on the allowed momentum cuts, and the inverse of the correlation length for operators carrying quantum numbers $\left(Q, S_{z}\right)=(0,0)$ as computed from the MPS transfer matrix spectrum. In the infinite bond-dimension limit, the latter quantity is a proxy for the gap to excitations with $S_{z}=0$ [56,76]; we present data for only a single bond dimension, but nevertheless a comparison of this inverse correlation length across parameter space can indicate which phases are likely to have a spin-singlet gap. All three quantities would be independent of $\theta$ in the limit of a very wide cylinder; here we see substantial variation, but at each $\theta$ the qualitative behavior as $U / t$ is varied remains essentially the same.

Most notably, the chiral order parameter is nonzero in a region of roughly constant width; furthermore, if for each $\theta$ we find the maximum value of the chiral order parameter versus $U / t$, these maxima vary with $\theta$ by only about $1 / 3$ of the maximum at $\theta=0$. The comparison between the three figures also reveals behavior for all $\theta$ that is in good agreement with what we found with periodic boundary conditions. In particular, the degree of short-range magnetic ordering rapidly increases at the right edge of the chiral phase, and furthermore the chiral phase appears to be strongly gapped, consistent with the analysis of central charge.

\section{B. YC6}

We next present data for the YC6 cylinder, which is the largest, and thus presumably the least impacted by finitesize effects, of those we study; this cylinder has the potential drawback that the MPS bond dimension required to achieve a given level of precision scales exponentially with $L$, so the simulations are less converged than for smaller cylinders, but we find that the qualitative behavior of the system is nevertheless clear.

The YC6 cylinder is notable not just because it is the widest of those we study but also because, as we show now, it has topologically degenerate ground states in two different momentum sectors. Because we employ a mixed realand momentum-space basis, we can initialize the DMRG with states in different sectors of momentum around the cylinder per unit length [77], $k$, and thus separately find the ground state in each sector. On the YC4 cylinder the ground state always lies in the $k=0$ sector, but for the YC6 cylinder we observe low-lying states in two different momentum sectors, $k=0$ and $k=\pi$. The relative energy difference between the ground states in the two sectors is shown in Fig. 7(a). There are three apparent regimes of behavior: at low $U$, the $k=0$ sector is clearly the ground state; at intermediate $U$, the two sectors become close in energy, and the difference decreases with increasing bond dimension; at high $U$, the $k=\pi$ sector becomes the ground state, though again the relative difference in energy decreases with increasing bond dimension.

The low- $U$ phase is expected to be metallic, with central charge $c=10[56,60]$. Finite entanglement scaling indeed suggests that the phase is gapless [56], though an accurate measurement of the central charge would require a bond dimension currently inaccessible to us, on the order of 50 000. (Extremely high entanglement in the low- $U$ region leads to very large DMRG truncation error, on the order of $10^{-4}$, even with $\chi \sim 10000$.) The high- $U$ phase should be
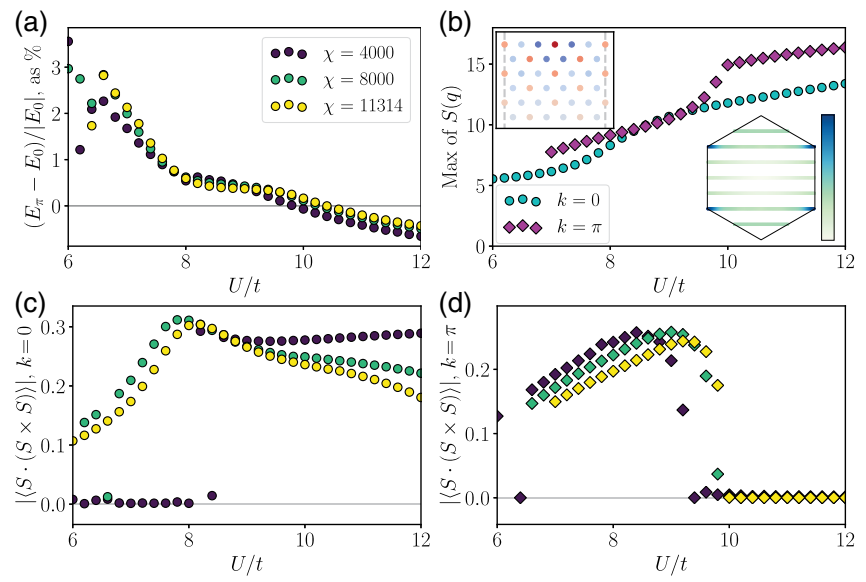

FIG. 7. Results for the YC6 cylinder. (a) Relative energy (percent difference) between ground states in the symmetry sectors with $k=\pi$ and $k=0$ around each ring. (b) Maximum value of the spin structure factor for the two momentum sectors. The insets show (lower right) the high- $U$ spin structure factor for the $k=\pi$ sector, with peaks at the $K^{ \pm}$points as expected for $120^{\circ}$ magnetic ordering, and (upper left) the corresponding real-space $\langle\mathbf{S} \cdot \mathbf{S}\rangle$ correlations to a chosen point (center on the top). (c) Chiral order parameter for the $k=0$ ground state. (d) Chiral order parameter for the $k=\pi$ ground state. 
the one-dimensional descendant of the two-dimensional $120^{\circ}$ Néel ordered phase, and indeed, at approximately the same value of $U / t$ where the $k=\pi$ sector becomes the ground state, there is a rapid increase in the peak height of the spin structure factor in the $k=\pi$ sector, as shown in Fig. 7(b). In this phase, we observe the expected peaks in the structure factor at the corners of the Brillouin zone (lower right inset) and short-range spin ordering in the realspace spin-spin correlations (upper left inset).

The intermediate phase, for $U / t \approx 8$ to $U / t \approx 10$, is the region where the relative energy difference between the two momentum sectors is small and approximately constant; the spin structure factors are also approximately equal. We identify the transition to the right by the onset of the aforementioned spin ordering. To the left, the transition can be observed by the $k=0$ sector becoming the sole ground state and from the transition in that sector to a metallic phase; as we show in the Supplemental Material [56], the latter can be seen qualitatively from the entanglement spectrum and finite entanglement scaling - the low- $U$ phase appears gapless, while the intermediate phase is likely gapped.

As with the YC4 cylinder, spontaneous breaking of timereversal symmetry leads to a nonzero value of the chiral order parameter in the metallic and intermediate phases, as shown for the two momentum sectors in Figs. 7(c) and 7(d), though in the metal we would expect the symmetry to be restored at larger bond dimensions. In the $k=\pi$ sector, which is the true ground state for high $U$, the chiral order parameter rapidly vanishes at the spin-ordering transition. In the $k=0$ sector, the chirality does not seem to drop abruptly to zero; however, as can be seen in Fig. 7(c), the chirality does rapidly decrease with increasing bond dimension for $U \gtrsim 10$.

We can again acquire more information about the full two-dimensional model by performing adiabatic flux insertion to scan the allowed momentum cuts through the full Brillouin zone; we perform the flux insertion using the $k=\pi$ ground state as the initial state with $\theta=0$, and we perform all computations with $\chi=8000$. Although the bond dimension is twice that used for YC4 flux insertion, the results are much less converged. Nevertheless, some features can be captured at least qualitatively, as shown in Fig. 8. In particular, there is a chiral phase for all $\theta$, which has weak local magnetic order and a sizable spin-singlet gap (indicated by the inverse correlation length). The chiral region extending to higher $U$ around $\theta=2 \pi$ is likely an artifact of the finite bond dimension: all local properties at $2 \pi$ flux are essentially identical to those of the $k=0$ ground state with periodic boundaries, and as noted above, the chiral order parameter is far from converged at $\chi=$ 8000 above $U \gtrsim 10$.

\section{YC5}

The YC4 and YC6 phase diagrams discussed above are qualitatively similar; both show a chiral intermediate phase
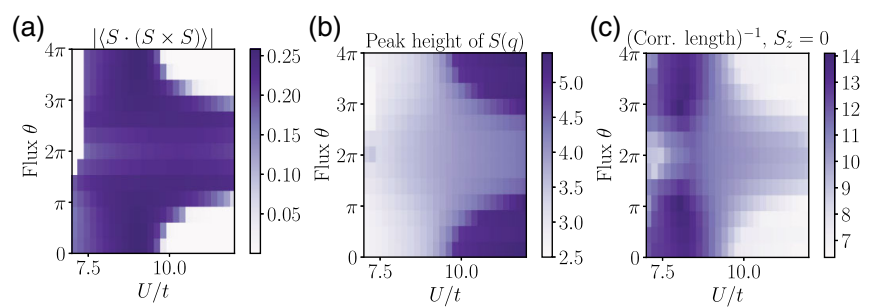

FIG. 8. YC6 cylinder with flux insertion $\theta$, for $\chi=8000$. (a) Absolute value of the chiral order parameter. (b) Maximum of $\left\langle S_{z} S_{z}\right\rangle$ structure factor on allowed momentum cuts in the Brillouin zone. (c) Inverse correlation length for excitations with $S_{z}=0$.

in the vicinity of $U / t=10$, which is present regardless of the twisting of the boundary conditions. The same is not true for the YC5 cylinder-with periodic boundary conditions, $\theta=0$, there is no spontaneous time-reversal symmetry breaking for any $U$. However, when we perform flux insertion, we find that the chiral intermediate phase does still exist, for $\pi \lesssim \theta \lesssim 3 \pi$ and $8 \lesssim U / t \lesssim 10$. This is shown in Fig. 9.

To understand these data, it is important to note that, unlike for YC4 and YC6, we use a two-ring unit cell; for an odd-circumference cylinder, we must use an even number of rings in order to initialize the DMRG simulation with a product state that is half-filled both for spin up and spin down. The two-ring unit cell allows the ground state to break translation symmetry along the cylinder, which
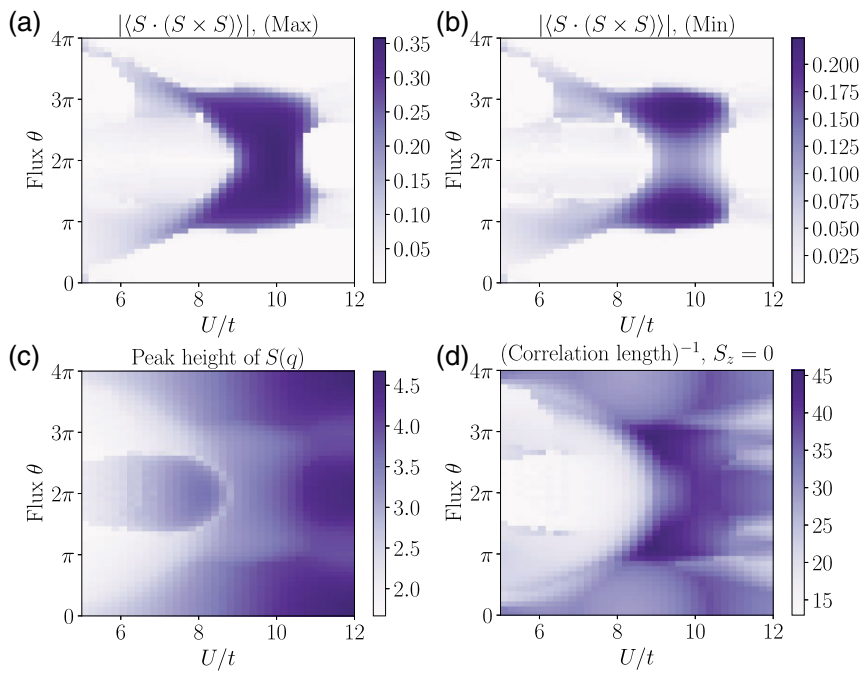

FIG. 9. YC5 cylinder with flux insertion $\theta$, for $\chi=4000$. (a) Absolute value of the chiral order parameter; at finite bond dimension, the translation symmetry is broken along the cylinder, and here we show the larger of the chiral order parameters between the two distinct rings. The chiral phase exists at intermediate $U$ when $\theta$ is approximately in the range $\pi-3 \pi$. (b) The smaller of the two chiral order parameters. (c) Maximum of the $\left\langle S_{z} S_{z}\right\rangle$ structure factor in the Brillouin zone. (d) Inverse correlation length for excitations with $S_{z}=0$. 
indeed occurs; this symmetry breaking is expected even in a spin liquid phase [78]. Figures 9(a) and 9(b) show the chiral order parameter on the two rings of the unit cell. As shown in Fig. 10(b), the degree of symmetry breaking decreases as the MPS bond dimension used in running DMRG is increased, though it appears that even at infinite bond dimension the symmetry will remain broken.

The chiral phase observed for YC5 seems to be the same as that found in YC4 and YC6 even if it does not extend through all boundary condition twists $\theta$. This is partially confirmed by considering the peak height of the $\left\langle S_{z} S_{z}\right\rangle$ structure factor and the inverse correlation length for excitations with $S_{z}=0$, shown in Figs. 9(c) and 9(d), respectively. As with YC4 and YC6, the chiral phase has a degree of short-range spin ordering which is intermediate between that of the metal and that of the high- $U$ phase and also has the largest spin-singlet gap of any region of the phase diagram. We also show below, in Sec. IV, that this chiral phase shows the same signatures of the topological chiral spin liquid as do the YC4 and YC6 phases.

As evidence for the existence of the chiral phase in the full two-dimensional model, the YC5 results are somewhat ambiguous. Neither $\theta=0$, for which there is no chiral phase, nor $\theta=2 \pi$, for which the chiral phase exists, is a priori "better" or more representative of the two-dimensional model. However, further insight can be gleaned by understanding the effect of the twisted boundaries on the spin degrees of freedom that are the relevant ones for a spin liquid phase. Indeed, we believe that the $\theta=2 \pi$ boundary conditions turn out to be the more representative ones.

In particular, we can look at the strength of $\left\langle S_{z} S_{z}\right\rangle$ correlators on bonds between adjacent sites; the results are shown for four bond dimensions up to $\chi=11314$ for $U / t=10$ in Figs. 10(c) and 10(d). Evidently, for flux near $\theta=0$, there is huge anisotropy, with spin correlations much stronger on bonds around the cylinder circumference than for diagonal ones. As the flux increases from zero, the anisotropy continuously decreases and shows only a change in slope upon entering the chiral phase; the anisotropy is smallest precisely where the chiral order parameter is largest. Assuming that the true intermediate phase of the two-dimensional model does not break the model's $C_{3}$ rotation symmetry, the $\theta$ for which the YC5 cylinder exhibits a chiral phase are precisely those in which the symmetry of the spin correlations is most two-dimensional. We also test this explanation by explicitly adding anisotropy to the model to weaken the bonds around the cylinder circumference; indeed, with the hopping strength on these bonds reduced by $10 \%$, a chiral phase appears even at zero flux [56].

\section{YC3}

The YC3 cylinder is the smallest, and thus presumably least representative of the two-dimensional model, of all those we study; we nevertheless include our data for (a)
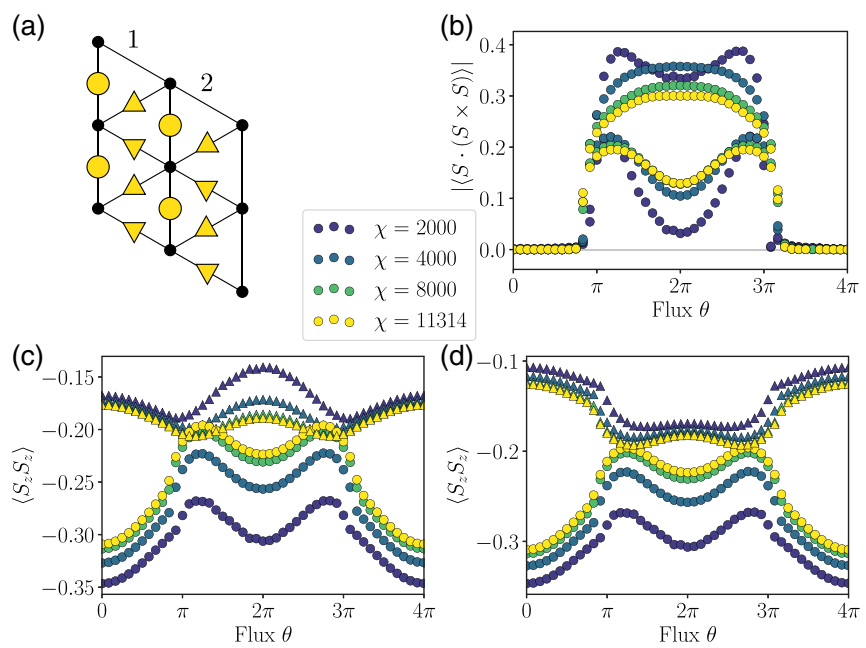

FIG. 10. Flux insertion for YC5 cylinder with $U / t=10$, for a range of bond dimensions. (a) As noted in the text, a two-ring unit cell allows for translation symmetry breaking. Here we label the distinct rings of the cylinder in the unit cell and the three bonds in each ring that may have different $\left\langle S_{z} S_{z}\right\rangle$ correlations. (b) Chiral order parameter on ring 1 (upper curves) and ring 2 (lower curves) of the unit cell. It appears that the lower curve is converged for the largest $\chi$, while the upper one is not, but it does not appear that the two will become equal even in the infinite $\chi$ limit. (c) $\left\langle S_{z} S_{z}\right\rangle$ for nearest-neighbor bonds on ring 1 as shown in (a); the symbol for each data point indicates that it corresponds to the bond labeled by that symbol in (a). We do not show the strength of the down-triangle bond because for each $\chi$ and $\theta$ it is equal to that of the up-triangle bond to better than one part in $10^{8}$. In the 2D model, all three bonds are equivalent; on the YC cylinder, the vertical bonds are inequivalent to the two diagonal ones. (d) $\left\langle S_{z} S_{z}\right\rangle$ for ring 2.

completeness. With periodic boundaries, $\theta=0$, we find much the same behavior as for YC4 and YC6, with an intermediate chiral phase between a metallic phase and a short-range magnetically ordered one. As partial evidence, we show the chiral order parameter versus $U / t$ in Fig. 11(a). Note that, as with YC5, we use a larger unit cell (in this case, four rings) and find that for finite bond dimension the model has only a two-ring translation symmetry; in the figure, the two symbols for each bond dimension show the chiral order parameter on the two distinct rings.

With flux insertion, the behavior is quite different, and, as we show in Fig. 11(b), the chirality vanishes for $\pi \lesssim \theta \lesssim 3 \pi$, essentially the opposite of the behavior observed for YC5. In Figs. 11(c) and 11(d), we also show the peak height of the spin structure factor and the inverse correlation length for excitations with $S_{z}=0$. The relationship between these quantities and the chirality is quite different from what we observe for all three cylinder geometries discussed above, so it is not clear that the chiral phase observed here corresponds to the one found for the larger cylinders. Additional data are available in the Supplemental Material [56]. 

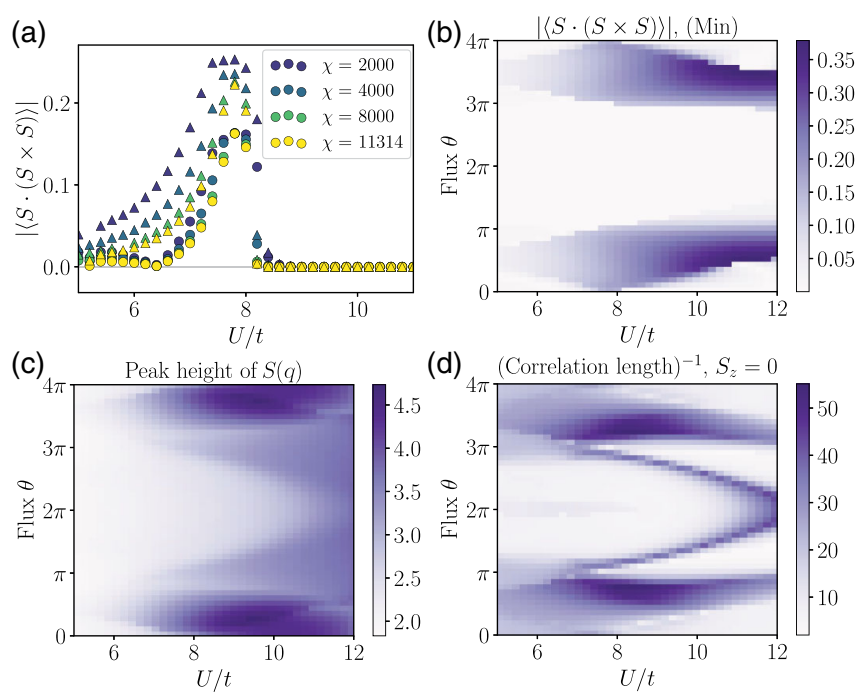

FIG. 11. Results for the YC3 cylinder. (a) Chiral order parameter on each of two rings (circle and triangle symbols, respectively), plotted versus $U / t$ for a range of bond dimensions. The behavior is qualitatively similar to that of YC4 and YC6. (b) Chiral order parameter versus $U / t$ and flux insertion $\theta$, for $\chi=4000$. Here we show just the smaller of the chiral order parameters on the two rings, but the qualitative behavior is essentially identical at this bond dimension. (c) Peak height of the $\left\langle S_{z} S_{z}\right\rangle$ structure factor in the Brillouin zone. (d) Inverse correlation length for excitations with $S_{z}=0$.

\section{E. XC4}

Finally, we place the model on the XC4 cylinder, which is the second smallest cylinder after YC3 [79]. With periodic boundaries, we find very weak time-reversal symmetry breaking for all $U / t$, which decreases with increasing bond dimension; the chiral order parameter is shown in Fig. 12(a). The extrapolation to infinite bond dimension is not entirely clear, but it is likely that the true ground state preserves the symmetry.

With flux insertion, we find that a chiral phase again appears, as shown in Fig. 12(b). We also show the peak height of the $\left\langle S_{z} S_{z}\right\rangle$ structure factor and the inverse correlation length for excitations with $S_{z}=0$, in Figs. 12(c) and 12(d), respectively. As with YC3, the $\mathrm{XC} 4$ cylinder does not show the clear relation between the three quantities that we found for YC4-6. However, like with YC5, the chirality appears near where the nearest-neighbor spin-spin correlations are most isotropic. In the high- $U$, midflux region [with a large spin-singlet gap in Fig. 12(d)], the diagonal bonds are much stronger than the horizontal ones, whereas in the rest of the phase diagram the opposite is true; the chirality is strongest precisely on the border between these two regions. Furthermore, the anisotropy is much larger in the region with exactly zero chirality than in the region where the chirality likely extrapolates to zero but is nonzero at finite bond dimension.
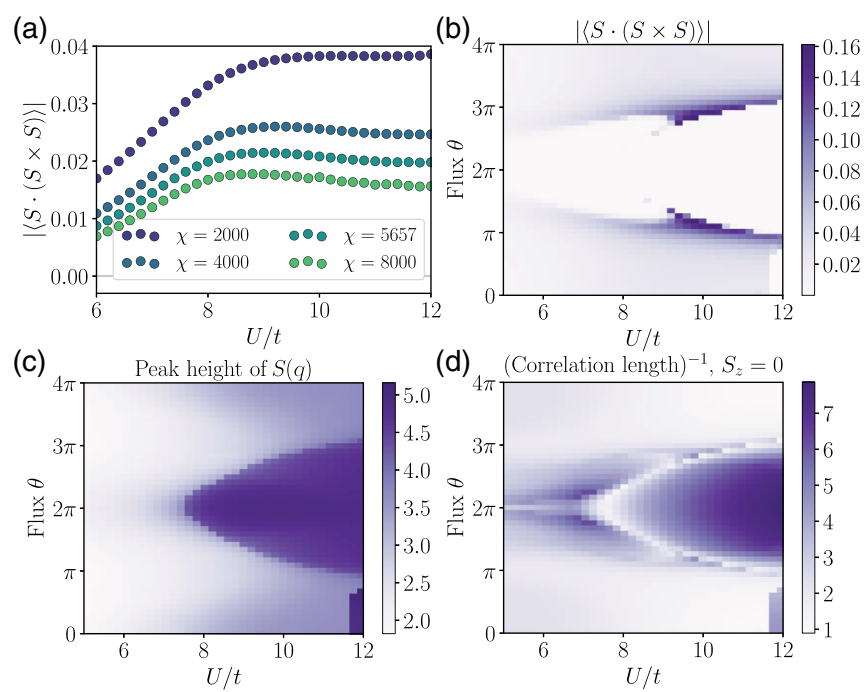

FIG. 12. Results for the XC4 cylinder. (a) Chiral order parameter versus $U / t$ for a range of bond dimensions, with periodic boundary conditions. The order parameter likely extrapolates to zero. (b) Chiral order parameter with flux insertion. (c) Maximum of the $\left\langle S_{z} S_{z}\right\rangle$ structure factor on allowed momentum cuts in the Brillouin zone. (d) Inverse correlation length for excitations with $S_{z}=0$.

\section{IDENTIFICATION AS A CHIRAL SPIN LIQUID}

We have demonstrated, for both the YC4 and YC6 cylinders, the existence of an intermediate phase which is nonmagnetic and which breaks time-reversal symmetry; we have also demonstrated that the phase is gapped for YC4 and likely gapped for YC6. We have furthermore observed this same phase for the YC5 cylinder for a range of twisted boundary conditions, and we have observed some similar behavior for the YC3 and XC4 cylinders. We now show that the chiral phase observed on the YC4-6 cylinders can, in fact, be identified as a chiral spin liquid (CSL) $[48,65]$.

A CSL is a topological phase with four degenerate ground states on the infinite cylinder [80]. Each minimally entangled ground state [81] spontaneously breaks timereversal $(T)$ and parity $(P)$ symmetries, as indicated by a nonzero value of the chiral scalar order parameter; the two possible chiralities account for a twofold degeneracy in the ground state manifold, which could be lifted by a $P$, $T$-breaking perturbation such as a magnetic field.

The remaining degeneracy is topological and is robust to such perturbations; the two topologically degenerate sectors, called the trivial and semion sectors, are distinguished by the respective absence or presence of a pair of semionic spinons, fractional excitations that carry spin $1 / 2$ but no charge, separated to the ends of the cylinder at $\pm \infty[80,82]$. In a pure spin system, insertion of $2 \pi$ flux creates a pair of spinons and separates them to the ends of the cylinder, thus exchanging the two ground states and also pumping a net spin of exactly $1 / 2$ across any cut through the cylinder; this latter property indicates that the CSL has a spin Chern 
number of $1 / 2$ and a corresponding quantized spin Hall conductance $[83,84]$.

In the Hubbard model, insertion of $2 \pi$ spin flux imposes antiperiodic boundary conditions on the cylinder, since $e^{2 \pi i S^{z}}=-1$. The Hamiltonian is thus modified by $2 \pi$ flux insertion, so that the question of whether the two ground state sectors are exchanged under flux insertion, as they are in a spin-model CSL, is ill defined; instead, $2 \pi$ flux insertion converts between one sector of the original Hamiltonian (with periodic boundaries) and the opposite sector of the Hamiltonian with antiperiodic boundaries, which should still lead to the same quantized spin pumping as for a spin model.

Each ground state of a CSL has a chiral edge mode with a universal low-lying spectrum; when the state is placed on an infinite cylinder, this edge spectrum appears in the entanglement spectrum for a cut between rings of the cylinder [85-87]. The edge modes are described by a chiral $S U(2)_{1}$ Wess-Zumino-Witten (WZW) conformal field theory [88]; labeling them by spin and momentum quantum numbers $[56,89,90]$, for a given spin the number of levels at successive discrete momenta around the cylinder follows the counting $(1,1,2,3,5, \ldots)[91]$. The spectrum is degenerate under $s_{z} \rightarrow-s_{z}$, where $s_{z}$ is the spin quantum number of the entanglement level; the spin quantum numbers are integers in the trivial sector and half-integers in the semion sector, leading to twofold degeneracy of the spectrum in the latter case.

We observe all of these signatures of the CSL phase. On the YC6 cylinder, we have already identified above two nearly degenerate low-lying states, in the $k=0$ and $k=\pi$ momentum sectors; within each sector, by initializing the DMRG with different product states, we are able to converge to both chiralities (see Supplemental Material [56]), thus finding all four degenerate ground states. The chiral order parameter in each sector, indicative of timereversal and parity symmetry breaking, has already been shown above in Figs. 7(c), 7(d), and 8(a); note that these figures show the absolute value of the order parameter, which is independent of the chirality to which the DMRG spontaneously converges.

The spin- and momentum-resolved entanglement spectra for the ground states in the two sectors are shown in Fig. 13(a), where we exclude levels corresponding to charge fluctuations between rings of the cylinder in order to highlight the spin degrees of freedom. Both spectra show the expected WZW level counting in the low-lying states for those momenta where the low-lying states can be distinguished from the continuum, and the spin quantum numbers of the entanglement levels are integer for the (a)

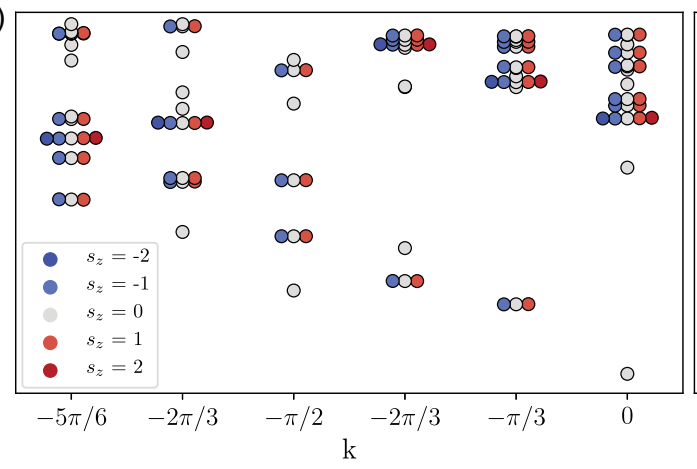

(b)

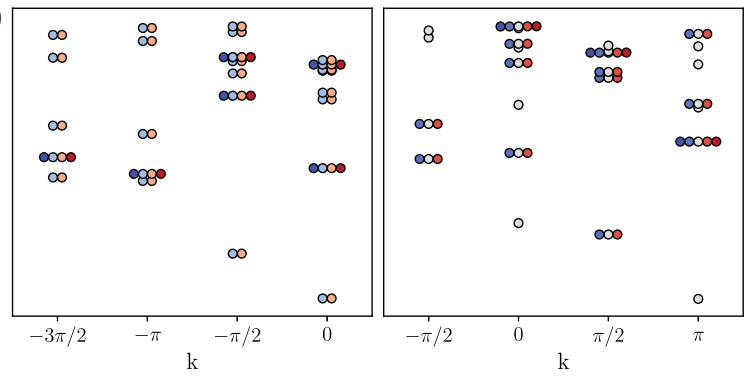

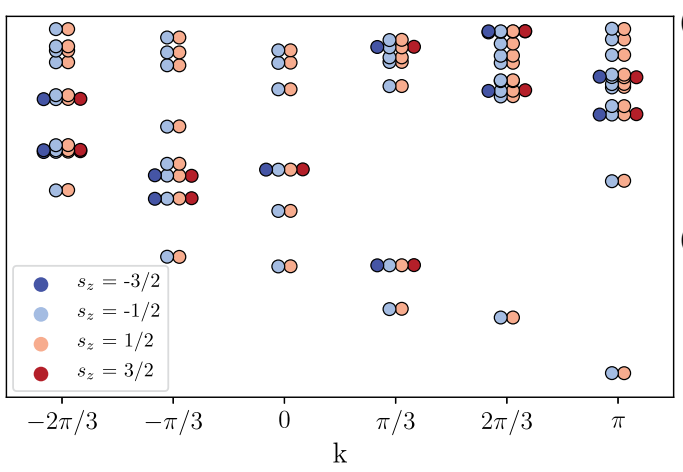
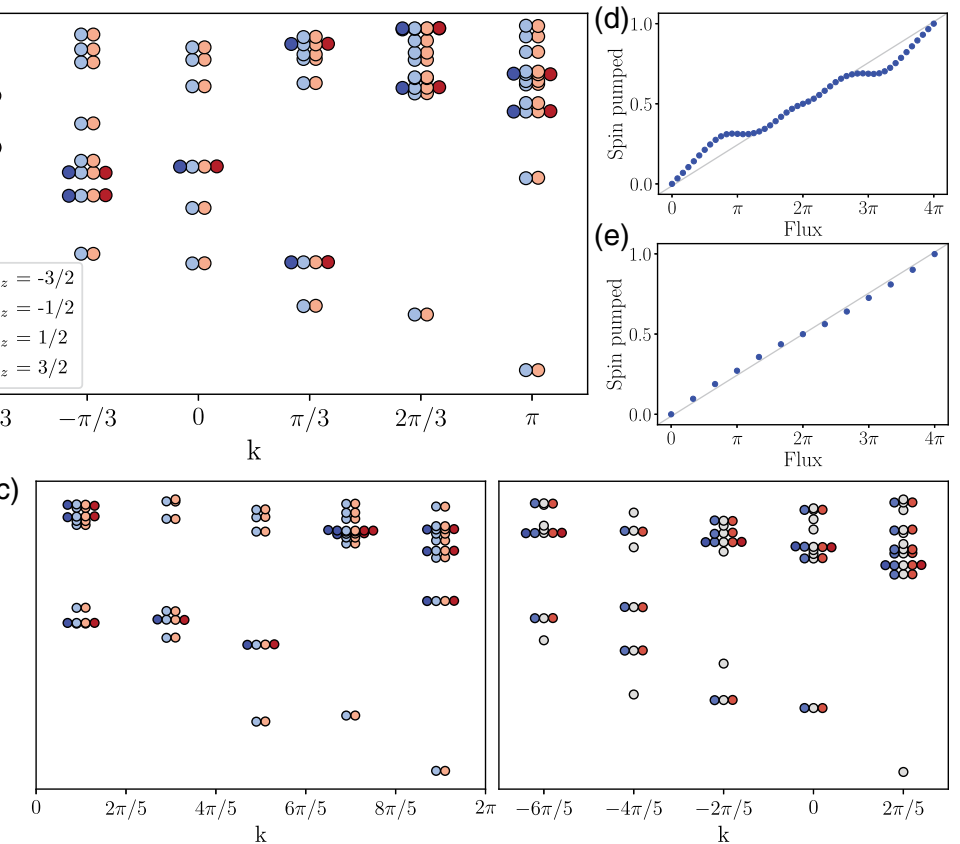

FIG. 13. (a) Momentum- and spin-resolved entanglement spectrum for the YC6 cylinder in the intermediate phase, for the ground state in the $k=0$ (left) and $k=\pi$ (right) sectors; these correspond to the trivial and semion sectors of a CSL, respectively. Insertion of $2 \pi$ flux interchanges the two topological sectors, though as discussed in the text there is a subtlety due to working with a fermion model. (b) Momentum- and spin-resolved entanglement spectrum for the YC4 cylinder, with periodic boundaries at $U / t=10.2$ (left) and with $2 \pi$ flux inserted at $U / t=11.6$ (right), corresponding to the highest chirality in each of the two topological sectors. (c) Entanglement spectrum for YC5 with $2 \pi$ flux, $U / t=10$, between two-ring unit cells (left) and between the rings in the unit cell (right), again corresponding to the two topological sectors. (d) Spin pumping as a function of flux insertion in the intermediate phase for YC4 $(U / t=10)$ and (e) for YC6 $(U / t=9)$. 
$k=0$ ground state and half-integer for $k=\pi$, allowing us to identify the low-lying states in the two momentum sectors with the trivial and semion topological sectors, respectively.

Alternatively, $2 \pi$ flux insertion should convert between the two topological sectors. We already noted in Sec. III B above that indeed the local properties like spin-spin correlations and the chiral order parameter look nearly identical between the $k=\pi$ sector with $2 \pi$ flux and the $k=0$ sector with periodic boundaries, which is consistent with this picture. (In principle, these should also be equal to the local properties of the $k=\pi$ sector with periodic boundaries, but that may not be true at finite bond dimension, and equality will be weakly violated even in the true ground state due to the finite circumference of the cylinder.) In the Supplemental Material [56], we show the equivalent of Fig. 13(a) with the $k=0$ entanglement spectrum replaced by the $k=\pi$ spectrum with $2 \pi$ flux, and evidently it is nearly identical.

To see the equivalent of Fig. 13(a) for the YC4 cylinder, because we find only one ground state sector, with $k=0$, we must use the flux insertion method. In Fig. 13(b), we show the spin- and momentum-resolved entanglement spectrum for $\mathrm{YC} 4$ in the $k=0$ sector, with periodic boundaries at $U / t=10.2$ and with $\theta=2 \pi$ at $U / t=11.6$; as shown in Fig. 6(a), these values of $U / t$ are each at the peak of the chiral order for their respective amounts of flux insertion, $\theta$. For the YC5 cylinder, as with YC4 we find a ground state only in the $k=0$ sector, although with two rings per unit cell, this sector includes both $k=0$ and $k=\pi$ per ring. In this case, however, we cannot observe both topological sectors by looking at $\theta=0$ and $2 \pi$, since the chiral phase exists only for $\pi \lesssim \theta \lesssim 3 \pi$. Instead, we make use of the fact that, for any cylinder with an odd number of spin $1 / 2$ per ring, translation along the cylinder converts between topological sectors [78], so that we can just consider a single wave function and examine its entanglement spectrum both between two-ring unit cells and between the two rings in the unit cell; the result is shown in Fig. 13(c).

With flux insertion, we also observe the quantized spin Hall effect, as shown for the YC4 and YC6 cylinders at $U / t=10$ and 9, respectively, in Figs. 13(d) and 13(e), with a pumping of exactly spin $1 / 2$ per $2 \pi$ flux insertion. For YC6, for which the chiral order is roughly constant at $U / t=9$, the flux insertion proceeds at a constant rate. For $\mathrm{YC}$, the shifting boundary of the chiral phase with flux insertion causes some deviation, but the qualitative behavior is the same.

\section{DISCUSSION}

By employing the DMRG method to study the triangular lattice Hubbard model on infinite cylinders in a mixed realand momentum-space basis, we observe that the model exhibits three phases: a metallic phase, a nonmagnetic insulating phase, and a magnetically ordered phase. While the nature of the intermediate phase depends on the precise boundary conditions used, with flux insertion through the cylinder we find that for each cylinder geometry there is a region with spontaneous time-reversal symmetry breaking, as indicated by a nonzero chiral order parameter. In particular, this chiral intermediate phase exists for all values of flux insertion for the YC4 and YC6 cylinders and for a large range of flux for the YC5 cylinder; the YC5 chiral intermediate phase appears precisely for those amounts of flux insertion for which spin-spin correlations are most consistent with the symmetries of the twodimensional lattice.

Furthermore, we show for the YC4, YC5, and YC6 cylinders that the chiral phase shows the characteristic entanglement spectrum of a CSL with two topologically degenerate ground state sectors, and for YC4 and YC6 we demonstrate a fractionally quantized spin Hall effect. The phase additionally appears to be gapped. Along with the nonzero chiral order parameter, this evidence strongly suggests that the nonmagnetic insulating phase is, in fact, a chiral spin liquid. Our results provide, to our knowledge, the first clear demonstration of a chiral spin liquid in a timereversal symmetric model of itinerant fermions.

The apparent gapped nature of the spin liquid in our simulations is consistent with the thermal conductivity measurements on $\kappa$-(BEDT-TTF $)_{2} \mathrm{Cu}_{2}(\mathrm{CN})_{3}$ reported in Ref. [9]; some recent experiments [14,15] also suggest gapped thermal conductivity in $\mathrm{EtMe}_{3} \mathrm{Sb}\left[\mathrm{Pd}(\mathrm{dmit})_{2}\right]_{2}$, although the implications for a possible spin liquid ground state are disputed [16]. On the other hand, our conclusions about the nature of the spin liquid do not agree with those of past studies of this model using the DMRG method: the study on the two-leg ladder finds a gapless spin liquid phase [45], while the DMRG study on a finite XC6 cylinder finds an intermediate phase that appears gapped but with a rapidly decaying chiral-chiral correlation function [47]. The two-leg ladder study uses a modified Hamiltonian with some longer-range interactions, so the disagreement on the nature of the spin liquid is not surprising. The discrepancy with the XC6 finite cylinder study is more difficult to explain. One possibility is that, as with the XC4 and YC5 cylinders in our study, the XC6 cylinder would exhibit a chiral phase after flux insertion; we are not able to reach high enough bond dimension to converge the XC6 cylinder, and thus are unfortunately not able to test this possibility.

It is also useful to briefly consider other candidates for the intermediate phase. In particular, it is worth investigating the possibility of the intermediate phase being a Dirac spin liquid (DSL), both because there has recently been evidence in support of a DSL in frustrated spin models $[92,93]$ and because the CSL can be derived by gapping out the Dirac cones in a DSL, so that one might imagine a DSL in two dimensions becoming a CSL due to either finite cylinder circumference or finite bond dimension. The first 
scenario is difficult to rule out, given that the CSL would still be the true ground state up to some cylinder circumference which could be much larger than what is accessible, but there is also no particular evidence from our data to support this scenario. The second scenario we do rule out, by analyzing the low-lying excitation spectrum using the MPS transfer matrix spectra; this analysis is described in detail in the Supplemental Material [56].

If the CSL is indeed the ground state in the full twodimensional triangular lattice Hubbard model, in real materials well described by this model we would expect regions of both possible chiralities to coexist, with a finitetemperature phase transition to long-range chiral order at a temperature of the same order of magnitude as the chiral domain wall tension, possibly reduced due to entropy from the gapless edge modes located at the domain walls. We measure this domain wall tension for the YC4 cylinder by finding an optimized composite wave function that transitions from the ground state with one chirality to the ground state with the other, and we find a domain wall tension of approximately $0.0065 t$ per lattice constant; using estimates for $t$ for real materials [7], this evaluates to about $4 K \times k_{B}$ [56]. The corresponding phase transition may be related to the observed feature in the heat capacity, thermal conductivity, and magnetic relaxation rate at about $6 \mathrm{~K}$ in $\kappa$-(BEDT-TTF $)_{2} \mathrm{Cu}_{2}(\mathrm{CN})_{3}[8,9,94]$.

At very low temperatures in a single-domain sample, we would observe no longitudinal thermal transport, in agreement with experimental data $[9,14,15]$, and a quantized thermal Hall conductance, $K_{x y}=\left(\pi^{2} k_{B}{ }^{2} T\right) /(3 h)$; note that the latter is twice the value of the Majorana-like plateau recently reported in $\alpha-\mathrm{RuCl}_{3}[95,96]$. In the presence of time-reversal symmetry-breaking disorder, there would be regions of both possible chiralities with gapless edge modes between them. It would be interesting to further investigate the resulting behavior of the specific heat and the thermal conductivity in this scenario.

An applied magnetic field could, in principle, break the degeneracy between the two chiralities, but this effect is extremely small at experimentally accessible field strengths. If the magnetic flux through a triangle in the lattice is $\phi$, perturbation theory in $t / U$ gives a term $\left[24\left(t^{3} / U^{2}\right) \sin (\phi)(\mathbf{S} \cdot(\mathbf{S} \times \mathbf{S})) / \hbar^{3}\right]$ in the effective spin Hamiltonian; using our measured value for the chiral order parameter and estimated parameters for $\kappa$-(BEDT-TTF $)_{2} \mathrm{Cu}_{2}(\mathrm{CN})_{3}$ [7,97], in a $10 \mathrm{~T}$ field the energy splitting between ground states for the two chiralities is about $1 \mu \mathrm{eV}$ per lattice site, so at $1 \mathrm{~K}$ the favored chirality would be expected to be just $1 \%$ more prevalent. So it would not be surprising for experiments to see no significant effect for applied magnetic fields up to $10 \mathrm{~T}$ [8].

In addition to studying the chiral spin liquid phase, we also considered the transitions to the neighboring metallic and spin-ordered phases. For the YC4 cylinder in particular, for which our data are the most extensive, every quantity we compute, including correlation length, spin order, chiral order, and an estimate for the quasiparticle weight, shows no discontinuity at the metal-insulator transition, suggesting that it is second order. However, we are not aware of any field theory description of a direct metal to chiral spin liquid transition, and furthermore a recent experiment tuning across the metal-insulator transition by doping found the transition to be first order [98], so further study is very much warranted. Some possibilities include a weakly first-order transition in the full twodimensional model or the presence of a very small intermediate phase such as the aforementioned Dirac spin liquid; alternatively, a theory of this transition may simply be waiting to be discovered. Future numerical work focusing specifically on the metal-insulator transition will hopefully be able to resolve the exact nature of the transition, including finding the critical exponents if it is indeed continuous.

More generally, further theoretical work must address the question of whether the chiral phase we find on the cylinders we study indeed extrapolates to the full twodimensional model. Our results strongly support this conclusion: on the YC4 and YC6 cylinders, the chiral phase exists for a large range of $U / t$ independent of the flux insertion that scans the allowed momentum cuts through the full two-dimensional Brillouin zone, and furthermore, on the YC5 cylinder, the same phase appears when the twisted boundary conditions lead to spin correlations that approximately obey the symmetries of the full twodimensional lattice. In other words, the chiral spin liquid is always present in the model as a competing phase, and it seems to be favored in those situations that best represent the two-dimensional system. The existence of the chiral spin liquid in two dimensions could be further confirmed either by using larger circumferences, which would be computationally expensive, or by fully $2 \mathrm{D}$ methods such as projected entangled pair states (PEPS) $[99,100]$.

\section{ACKNOWLEDGMENTS}

We thank Bryan Clark, Yin-Chen He, Ryan Mishmash, Roger Mong, Frank Pollmann, Tomonori Shirakawa, Shigetoshi Sota, Senthil Todadri, Hiroshi Ueda, Ruben Verresen, and Seiji Yunoki for helpful conversations. We have also used the TenPy tensor network library [101], which includes contributions from M.P.Z., Mong, Pollmann, and others. Numerical computations were primarily performed using the Lawrencium cluster at Lawrence Berkeley National Laboratory. This work was funded by the U.S. Department of Energy, Office of Science, Office of Basic Energy Sciences, Materials Sciences and Engineering Division under Contract No. DE-AC02-05-CH11231 through the Scientific Discovery through Advanced Computing (SciDAC) program (KC23DAC Topological and Correlated Matter via Tensor Networks and Quantum Monte Carlo) (A. S., M. P.Z., and J.E. M.) and 
the Theory Institute for Materials and Energy Spectroscopies (TIMES) (J. M.). J. M. acknowledges support through DFG research fellowship No. MO 3278/1-1. Research at Perimeter Institute is supported in part by the Government of Canada through the Department of Innovation, Science and Economic Development Canada and by the Province of Ontario through the Ministry of Economic Development, Job Creation and Trade. Publication made possible in part by support from the Berkeley Research Impact Initiative (BRII) sponsored by the UC Berkeley Library.

[1] L. Balents, Spin Liquids in Frustrated Magnets, Nature (London) 464, 199 (2010).

[2] L. Savary and L. Balents, Quantum Spin Liquids: A Review, Rep. Prog. Phys. 80, 016502 (2017).

[3] Y. Zhou, K. Kanoda, and T.-K. Ng, Quantum Spin Liquid States, Rev. Mod. Phys. 89, 025003 (2017).

[4] P. W. Anderson, Resonating Valence Bonds: A New Kind of Insulator?, Mater. Res. Bull. 8, 153 (1973).

[5] D. A. Huse and V. Elser, Simple Variational Wave Functions for Two-Dimensional Heisenberg Spin-1/2 Antiferromagnets, Phys. Rev. Lett. 60, 2531 (1988).

[6] S. R. White and A. L. Chernyshev, Neél Order in Square and Triangular Lattice Heisenberg Models, Phys. Rev. Lett. 99, 127004 (2007).

[7] Y. Shimizu, K. Miyagawa, K. Kanoda, M. Maesato, and G. Saito, Spin Liquid State in an Organic Mott Insulator with a Triangular Lattice, Phys. Rev. Lett. 91, 107001 (2003).

[8] S. Yamashita, Y. Nakazawa, M. Oguni, Y. Oshima, H. Nojiri, Y. Shimizu, K. Miyagawa, and K. Kanoda, Thermodynamic Properties of a Spin-1/2 Spin-Liquid State in a $\kappa$-Type Organic Salt, Nat. Phys. 4, 459 (2008).

[9] M. Yamashita, N. Nakata, Y. Kasahara, T. Sasaki, N. Yoneyama, N. Kobayashi, S. Fujimoto, T. Shibauchi, and Y. Matsuda, Thermal-Transport Measurements in a Quantum Spin-Liquid State of the Frustrated Triangular Magnet $\kappa$-(BEDT-TTF $)_{2} \mathrm{Cu}_{2}(\mathrm{CN})_{3}$, Nat. Phys. 5, 44 (2009).

[10] T. Itou, A. Oyamada, S. Maegawa, M. Tamura, and R. Kato, Quantum Spin Liquid in the Spin-1/2 Triangular Antiferromagnet $\mathrm{EtMe}_{3} \mathrm{Sb}\left[\mathrm{Pd}(\mathrm{dmit})_{2}\right]_{2}$, Phys. Rev. B 77, 104413 (2008).

[11] T. Itou, A. Oyamada, S. Maegawa, M. Tamura, and R. Kato, ${ }^{13} \mathrm{C} N M R$ Study of the Spin-Liquid State in the Triangular Quantum Antiferromagnet $\mathrm{EtMe}_{3} \mathrm{Sb}\left[\mathrm{Pd}(\mathrm{dmit})_{2}\right]_{2}$, J. Phys. Conf. Ser. 145, 012039 (2009).

[12] M. Yamashita, N. Nakata, Y. Senshu, M. Nagata, H. M. Yamamoto, R. Kato, T. Shibauchi, and Y. Matsuda, Highly Mobile Gapless Excitations in a Two-Dimensional Candidate Quantum Spin Liquid, Science 328, 1246 (2010).

[13] T. Itou, A. Oyamada, S. Maegawa, and R. Kato, Instability of a Quantum Spin Liquid in an Organic TriangularLattice Antiferromagnet, Nat. Phys. 6, 673 (2010).

[14] J. M. Ni, B. L. Pan, B. Q. Song, Y. Y. Huang, J. Y. Zeng, Y. J. Yu, E. J. Cheng, L. S. Wang, D. Z. Dai, R. Kato, and S. Y. Li, Absence of Magnetic Thermal Conductivity in the
Quantum Spin Liquid Candidate $\mathrm{EtMe}_{3} \mathrm{Sb}\left[\mathrm{Pd}(\mathrm{dmit})_{2}\right]_{2}$, Phys. Rev. Lett. 123, 247204 (2019).

[15] P. Bourgeois-Hope, F. Laliberté, E. Lefrançois, G. Grissonnanche, S. R. de Cotret, R. Gordon, S. Kitou, H. Sawa, H. Cui, R. Kato, L. Taillefer, and N. DoironLeyraud, Thermal Conductivity of the Quantum Spin Liquid Candidate $\mathrm{EtMe}_{3} \mathrm{Sb}\left[\mathrm{Pd}(\mathrm{dmit})_{2}\right]_{2}$ : No Evidence of Mobile Gapless Excitations, Phys. Rev. X 9, 041051 (2019).

[16] M. Yamashita, Boundary-Limited and Glassy-like Phonon Thermal Conduction in $\mathrm{EtMe}_{3} \mathrm{Sb}\left[\mathrm{Pd}(\mathrm{dmit})_{2}\right]_{2}$, J. Phys. Soc. Jpn. 88, 083702 (2019).

[17] K. T. Law and P. A. Lee, $1 \mathrm{~T}-\mathrm{TaS}_{2}$ as a Quantum Spin Liquid, Proc. Natl. Acad. Sci. U.S.A. 114, 6996 (2017).

[18] N. Li, W. J. Chu, Q. Huang, Q. Chen, X. Y. Yue, X. Zhao, H. D. Zhou, and X.F. Sun, Observation of Itinerant Gapless Excitations in Quantum Spin Liquid $\mathrm{Na}_{2} \mathrm{BaCo}\left(\mathrm{PO}_{4}\right)_{2}$, arXiv:1911.11107.

[19] K. Y. Zeng, Long Ma, Y. X. Gao, Z. M. Tian, L. S. Ling, and L. Pi, NMR Evidence for Gapless Quantum Spin Liquid State in the Ideal Triangular-Lattice Compound $\mathrm{Yb}\left(\mathrm{BaBO}_{3}\right)_{3}$, arXiv:1912.03814.

[20] Y.-D. Li, Y. Shen, Y. Li, J. Zhao, and G. Chen, Effect of Spin-Orbit Coupling on the Effective-Spin Correlation in $\mathrm{YbMgGaO}_{4}$, Phys. Rev. B 97, 125105 (2018).

[21] Y. Shen, Y.-D. Li, H. C. Walker, P. Steffens, M. Boehm, X. Zhang, S. Shen, H. Wo, G. Chen, and J. Zhao, Fractionalized Excitations in the Partially Magnetized Spin Liquid Candidate $\mathrm{YbMgGaO}_{4}$, Nat. Commun. 9, 4138 (2018).

[22] O. I. Motrunich, Variational Study of Triangular Lattice Spin-1/2 Model with Ring Exchanges and Spin Liquid State in $\kappa-(\mathrm{ET})_{2} \mathrm{Cu}_{2}(\mathrm{CN})_{3}$, Phys. Rev. B 72, 045105 (2005).

[23] D. N. Sheng, O. I. Motrunich, and M. P. A. Fisher, Spin BoseMetal Phase in a Spin- $\frac{1}{2}$ Model with Ring Exchange on a TwoLeg Triangular Strip, Phys. Rev. B 79, 205112 (2009).

[24] T. Grover, N. Trivedi, T. Senthil, and P. A. Lee, Weak Mott Insulators on the Triangular Lattice: Possibility of a Gapless Nematic Quantum Spin Liquid, Phys. Rev. B 81, 245121 (2010).

[25] M. S. Block, D. N. Sheng, O. I. Motrunich, and M. P. A. Fisher, Spin Bose-Metal and Valence Bond Solid Phases in a Spin-1/2 Model with Ring Exchanges on a Four-Leg Triangular Ladder, Phys. Rev. Lett. 106, 157202 (2011).

[26] R. V. Mishmash, J. R. Garrison, S. Bieri, and C. Xu, Theory of a Competitive Spin Liquid State for Weak Mott Insulators on the Triangular Lattice, Phys. Rev. Lett. 111, 157203 (2013).

[27] R. Kaneko, S. Morita, and M. Imada, Gapless Spin-Liquid Phase in an Extended Spin 1/2 Triangular Heisenberg Model, J. Phys. Soc. Jpn. 83, 093707 (2014).

[28] W.-J. Hu, S.-S. Gong, W. Zhu, and D. N. Sheng, Competing Spin-Liquid States in the Spin- $\frac{1}{2}$ Heisenberg Model on the Triangular Lattice, Phys. Rev. B 92, 140403(R) (2015).

[29] Z. Zhu and S. R. White, Spin Liquid Phase of the $S=\frac{1}{2}$ $J_{1}-J_{2}$ Heisenberg Model on the Triangular Lattice, Phys. Rev. B 92, 041105(R) (2015).

[30] S.-S. Gong, W. Zhu, J.-X. Zhu, D. N. Sheng, and K. Yang, Global Phase Diagram and Quantum Spin Liquids in a Spin- $\frac{1}{2}$ Triangular Antiferromagnet, Phys. Rev. B 96, 075116 (2017).

[31] S. N. Saadatmand and I. P. McCulloch, Detection and Characterization of Symmetry-Broken Long-Range Orders 
in the Spin- $\frac{1}{2}$ Triangular Heisenberg Model, Phys. Rev. B 96, 075117 (2017).

[32] Z. Zhu, P. A. Maksimov, S. R. White, and A. L. Chernyshev, Topography of Spin Liquids on a Triangular Lattice, Phys. Rev. Lett. 120, 207203 (2018).

[33] J. Sahoo, D. Kochkov, B. K. Clark, and R. Flint, Classical Phase Diagram of the Stuffed Honeycomb Lattice, Phys. Rev. B 98, 134419 (2018).

[34] K. Riedl, R. Valentí, and S. M. Winter, Critical Spin Liquid versus Valence-Bond Glass in a Triangular-Lattice Organic Antiferromagnet, Nat. Commun. 10, 2561 (2019).

[35] A. H. MacDonald, S. M. Girvin, and D. Yoshioka, $t / U$ Expansion for the Hubbard Model, Phys. Rev. B 37, 9753 (1988).

[36] H. Morita, S. Watanabe, and M. Imada, Nonmagnetic Insulating States near the Mott Transitions on Lattices with Geometrical Frustration and Implications for $\kappa-(\mathrm{ET})_{2} \mathrm{Cu}_{2}(\mathrm{CN})_{3}$, J. Phys. Soc. Jpn. 71, 2109 (2002).

[37] B. Kyung and A.-M. S. Tremblay, Mott Transition, Antiferromagnetism, and d-Wave Superconductivity in TwoDimensional Organic Conductors, Phys. Rev. Lett. 97, 046402 (2006).

[38] P. Sahebsara and D. Sénéchal, Hubbard Model on the Triangular Lattice: Spiral Order and Spin Liquid, Phys. Rev. Lett. 100, 136402 (2008).

[39] L. F. Tocchio, A. Parola, C. Gros, and F. Becca, SpinLiquid and Magnetic Phases in the Anisotropic Triangular Lattice: The Case of $\kappa-(\mathrm{ET})_{2} \mathrm{X}$, Phys. Rev. B 80, 064419 (2009).

[40] T. Yoshioka, A. Koga, and N. Kawakami, Quantum Phase Transitions in the Hubbard Model on a Triangular Lattice, Phys. Rev. Lett. 103, 036401 (2009).

[41] H.-Yu. Yang, A. M. Läuchli, F. Mila, and K. P. Schmidt, Effective Spin Model for the Spin-Liquid Phase of the Hubbard Model on the Triangular Lattice, Phys. Rev. Lett. 105, 267204 (2010).

[42] A. E. Antipov, A. N. Rubtsov, M. I. Katsnelson, and A. I. Lichtenstein, Electron Energy Spectrum of the Spin-Liquid State in a Frustrated Hubbard Model, Phys. Rev. B 83, 115126 (2011).

[43] L. F. Tocchio, H. Feldner, F. Becca, R. Valentí, and C. Gros, Spin-Liquid versus Spiral-Order Phases in the Anisotropic Triangular Lattice, Phys. Rev. B 87, 035143 (2013).

[44] M. Laubach, R. Thomale, C. Platt, W. Hanke, and G. Li, Phase Diagram of the Hubbard Model on the Anisotropic Triangular Lattice, Phys. Rev. B 91, 245125 (2015).

[45] R. V. Mishmash, I. González, R. G. Melko, O. I. Motrunich, and M. P. A. Fisher, Continuous Mott Transition between a Metal and a Quantum Spin Liquid, Phys. Rev. B 91, 235140 (2015).

[46] K. Misumi, T. Kaneko, and Y. Ohta, Mott Transition and Magnetism of the Triangular-Lattice Hubbard Model with Next-Nearest-Neighbor Hopping, Phys. Rev. B 95, 075124 (2017).

[47] T. Shirakawa, T. Tohyama, J. Kokalj, S. Sota, and S. Yunoki, Ground-State Phase Diagram of the Triangular Lattice Hubbard Model by the Density-Matrix Renormalization Group Method, Phys. Rev. B 96, 205130 (2017).
[48] V. Kalmeyer and R. B. Laughlin, Equivalence of the Resonating-Valence-Bond and Fractional Quantum Hall States, Phys. Rev. Lett. 59, 2095 (1987).

[49] G. Baskaran, Novel Local Symmetries and ChiralSymmetry-Broken Phases in $S=(1 / 2$ Triangular-Lattice Heisenberg Model, Phys. Rev. Lett. 63, 2524 (1989).

[50] W.-J. Hu, S.-S. Gong, and D. N. Sheng, Variational Monte Carlo Study of Chiral Spin Liquid in Quantum Antiferromagnet on the Triangular Lattice, Phys. Rev. B 94, 075131 (2016).

[51] A. Wietek and A. M. Läuchli, Chiral Spin Liquid and Quantum Criticality in Extended $S=\frac{1}{2}$ Heisenberg Models on the Triangular Lattice, Phys. Rev. B 95, 035141 (2017).

[52] S. R. White, Density Matrix Formulation for Quantum Renormalization Groups, Phys. Rev. Lett. 69, 2863 (1992).

[53] S. R. White, Density-Matrix Algorithms for Quantum Renormalization Groups, Phys. Rev. B 48, 10345 (1993).

[54] S. Östlund and S. Rommer, Thermodynamic Limit of Density Matrix Renormalization, Phys. Rev. Lett. 75, 3537 (1995).

[55] U. Schollwöck, The Density-Matrix Renormalization Group in the Age of Matrix Product States, Ann. Phys. (Amsterdam) 326, 96 (2011).

[56] See Supplemental Material at http://link.aps.org/ supplemental/10.1103/PhysRevX.10.021042 for further details.

[57] S. Yan, D. A. Huse, and S. R. White, Spin-Liquid Ground State of the $S=1 / 2$ Kagome Heisenberg Antiferromagnet, Science 332, 1173 (2011).

[58] J. Motruk, M. P. Zaletel, R. S. K. Mong, and F. Pollmann, Density Matrix Renormalization Group on a Cylinder in Mixed Real and Momentum Space, Phys. Rev. B 93, 155139 (2016).

[59] G. Ehlers, S. R. White, and R. M. Noack, Hybrid-Space Density Matrix Renormalization Group Study of the Doped Two-Dimensional Hubbard Model, Phys. Rev. B 95, 125125 (2017).

[60] P. Francesco, P. Mathieu, and D. Sénéchal, Conformal Field Theory (Springer-Verlag, New York, 1997), https:// doi.org/10.1007\%2F978-1-4612-2256-9.

[61] I. Affleck, Quantum Spin Chains and the Haldane Gap, J. Phys. Condens. Matter 1, 3047 (1989).

[62] D. N. Sheng, O. I. Motrunich, S. Trebst, E. Gull, and M. P. A. Fisher, Strong-Coupling Phases of Frustrated Bosons on a Two-Leg Ladder with Ring Exchange, Phys. Rev. B 78, 054520 (2008).

[63] S. D. Geraedts, M. P. Zaletel, R. S. K. Mong, M. A. Metlitski, A. Vishwanath, and O. I. Motrunich, The Half-Filled Landau Level: The Case for Dirac Composite Fermions, Science 352, 197 (2016).

[64] X. G. Wen and Q. Niu, Ground-State Degeneracy of the Fractional Quantum Hall States in the Presence of a Random Potential and on High-Genus Riemann Surfaces, Phys. Rev. B 41, 9377 (1990).

[65] X. G. Wen, F. Wilczek, and A. Zee, Chiral Spin States and Superconductivity, Phys. Rev. B 39, 11413 (1989).

[66] C. Hubig, J. Haegeman, and U. Schollwöck, Error Estimates for Extrapolations with Matrix-Product States, Phys. Rev. B 97, 045125 (2018). 
[67] M. Capello, F. Becca, M. Fabrizio, S. Sorella, and E. Tosatti, Variational Description of Mott Insulators, Phys. Rev. Lett. 94, 026406 (2005).

[68] C. De Franco, L. F. Tocchio, and F. Becca, Metal-Insulator Transitions, Superconductivity, and Magnetism in the Two-Band Hubbard Model, Phys. Rev. B 98, 075117 (2018).

[69] L. Tagliacozzo, T. R. de Oliveira, S. Iblisdir, and J. I. Latorre, Scaling of Entanglement Support for Matrix Product States, Phys. Rev. B 78, 024410 (2008).

[70] F. Pollmann, S. Mukerjee, A. M. Turner, and J. E. Moore, Theory of Finite-Entanglement Scaling at OneDimensional Quantum Critical Points, Phys. Rev. Lett. 102, 255701 (2009).

[71] B. Pirvu, G. Vidal, F. Verstraete, and L. Tagliacozzo, Matrix Product States for Critical Spin Chains: Finite-Size versus Finite-Entanglement Scaling, Phys. Rev. B 86, 075117 (2012).

[72] P. Calabrese and J. Cardy, Entanglement Entropy and Quantum Field Theory, J. Stat. Mech. (2004) P06002.

[73] M. B. Hastings, An Area Law for One-Dimensional Quantum Systems, J. Stat. Mech. (2007) P08024.

[74] I. Arad, A. Kitaev, Z. Landau, and U. Vazirani, An Area Law and Sub-exponential Algorithm for $1 D$ Systems, arXiv:1301.1162.

[75] F. Pollmann, A. M. Turner, E. Berg, and M. Oshikawa, Entanglement Spectrum of a Topological Phase in One Dimension, Phys. Rev. B 81, 064439 (2010).

[76] V. Zauner, D. Draxler, L. Vanderstraeten, M. Degroote, J. Haegeman, M. M. Rams, V. Stojevic, N. Schuch, and F. Verstraete, Transfer Matrices and Excitations with Matrix Product States, New J. Phys. 17, 053002 (2015).

[77] M. P. Zaletel, Y.-M. Lu, and A. Vishwanath, Measuring Space-Group Symmetry Fractionalization in $\mathrm{Z}_{2}$ Spin Liquids, Phys. Rev. B 96, 195164 (2017).

[78] M. P. Zaletel and A. Vishwanath, Constraints on Topological Order in Mott Insulators, Phys. Rev. Lett. 114, 077201 (2015).

[79] Ideally, we would also consider the XC6 cylinder, but we are unable to reach a large enough bond dimension to converge the DMRG; at our largest accessible bond dimensions, there remains a strong symmetry-breaking effect from the orientation of the DMRG snake.

[80] X. G. Wen, Vacuum Degeneracy of Chiral Spin States in Compactified Space, Phys. Rev. B 40, 7387 (1989).

[81] Y. Zhang, T. Grover, A. Turner, M. Oshikawa, and A. Vishwanath, Quasiparticle Statistics and Braiding from Ground-State Entanglement, Phys. Rev. B 85, 235151 (2012).

[82] M. Oshikawa and T. Senthil, Fractionalization, Topological Order, and Quasiparticle Statistics, Phys. Rev. Lett. 96, 060601 (2006).

[83] S.-S. Gong, W. Zhu, and D. N. Sheng, Emergent Chiral Spin Liquid: Fractional Quantum Hall Effect in a Kagome Heisenberg Model, Sci. Rep. 4, 6317 (2015).

[84] A. G. Grushin, J. Motruk, M. P. Zaletel, and F. Pollmann, Characterization and Stability of a Fermionic $\nu=1 / 3$ Fractional Chern Insulator, Phys. Rev. B 91, 035136 (2015).

[85] A. Kitaev and J. Preskill, Topological Entanglement Entropy, Phys. Rev. Lett. 96, 110404 (2006).
[86] H. Li and F. D. M. Haldane, Entanglement Spectrum as a Generalization of Entanglement Entropy: Identification of Topological Order in Non-Abelian Fractional Quantum Hall Effect States, Phys. Rev. Lett. 101, 010504 (2008).

[87] X.-L. Qi, H. Katsura, and A. W. W. Ludwig, General Relationship between the Entanglement Spectrum and the Edge State Spectrum of Topological Quantum States, Phys. Rev. Lett. 108, 196402 (2012).

[88] X. G. Wen, Gapless Boundary Excitations in the Quantum Hall States and in the Chiral Spin States, Phys. Rev. B 43, 11025 (1991).

[89] M. P. Zaletel, R. S. K. Mong, and F. Pollmann, Topological Characterization of Fractional Quantum Hall Ground States from Microscopic Hamiltonians, Phys. Rev. Lett. 110, 236801 (2013).

[90] L. Cincio and G. Vidal, Characterizing Topological Order by Studying the Ground States on an Infinite Cylinder, Phys. Rev. Lett. 110, 067208 (2013).

[91] J. E. Moore and F. D. M. Haldane, Edge Excitations of the $\nu=$ Spin-Singlet Quantum Hall State, Phys. Rev. B 55, 7818 (1997).

[92] Y.-C. He, M. P. Zaletel, M. Oshikawa, and F. Pollmann, Signatures of Dirac Cones in a DMRG Study of the Kagome Heisenberg Model, Phys. Rev. X 7, 031020 (2017).

[93] S. Hu, W. Zhu, S. Eggert, and Y.-C. He, Dirac Spin Liquid on the Spin-1/2 Triangular Heisenberg Antiferromagnet, Phys. Rev. Lett. 123, 207203 (2019).

[94] Y. Shimizu, K. Miyagawa, K. Kanoda, M. Maesato, and G. Saito, Emergence of Inhomogeneous Moments from Spin Liquid in the Triangular-Lattice Mott Insulator $\kappa-(\mathrm{ET})_{2} \mathrm{Cu}_{2}(\mathrm{CN})_{3}$, Phys. Rev. B 73, 140407(R) (2006).

[95] Y. Kasahara, T. Ohnishi, Y. Mizukami, O. Tanaka, S. Ma, K. Sugii, N. Kurita, H. Tanaka, J. Nasu, Y. Motome, T. Shibauchi, and Y. Matsuda, Majorana Quantization and Half-Integer Thermal Quantum Hall Effect in a Kitaev Spin Liquid, Nature (London) 559, 227 (2018).

[96] T. Yokoi, S. Ma, Y. Kasahara, S. Kasahara, T. Shibauchi, N. Kurita, H. Tanaka, J. Nasu, Y. Motome, C. Hickey, S. Trebst, and Y. Matsuda, Half-Integer Quantized Anomalous Thermal Hall Effect in the Kitaev Material $\alpha-\mathrm{RuCl}_{3}$, arXiv:2001.01899.

[97] T. Komatsu, N. Matsukawa, T. Inoue, and G. Saito, Realization of Superconductivity at Ambient Pressure by Band-Filling Control in $\kappa$-(BEDT-TTF $)_{2} \mathrm{Cu}_{2}(\mathrm{CN})_{3}$, J. Phys. Soc. Jpn. 65, 1340 (1996).

[98] Y. Saito, R. Rösslhuber, A. Löhle, M. S. Alonso, M. Wenzel, A. Kawamoto, A. Pustogow, and M. Dressel, Bandwidth-Tuning from Insulating Mott Quantum Spin Liquid to Fermi Liquid via Chemical Substitution in $\kappa$ - $\left[(\mathrm{BEDT}-\mathrm{TTF})_{1-x}(\mathrm{BEDT}-\mathrm{STF})_{x}\right]_{2} \mathrm{Cu}_{2}(\mathrm{CN})_{3}$, arXiv: 1911.06766.

[99] F. Verstraete and J. I. Cirac, Renormalization Algorithms for Quantum-Many Body Systems in Two and Higher Dimensions, arXiv:cond-mat/0407066.

[100] P. Corboz, R. Orús, B. Bauer, and G. Vidal, Simulation of Strongly Correlated Fermions in Two Spatial Dimensions with Fermionic Projected Entangled-Pair States, Phys. Rev. B 81, 165104 (2010).

[101] J. A. Kjäll, M. P. Zaletel, R. S. K. Mong, J. H. Bardarson, and F. Pollmann, Phase Diagram of the Anisotropic Spin-2 XXZ Model: Infinite-System Density Matrix Renormalization Group Study, Phys. Rev. B 87, 235106 (2013). 